\title{
Character tables of Sylow $p$-subgroups of the Steinberg triality groups ${ }^{3} D_{4}\left(q^{3}\right)$
}

\author{
Yujiao Sun \\ School of Mathematics and Statistics, Beijing Institute of Technology, \\ Beijing 100081, PR China \\ E-mail: yujiaosun@bit.edu.cn
}

\begin{abstract}
We determine the character tables of Sylow $p$-subgroups $U$ of the Steinberg triality groups ${ }^{3} D_{4}\left(q^{3}\right)$, where $q$ is a power of an odd prime $p$.
\end{abstract}

Keywords: Character table, Sylow $p$-subgroup, Steinberg triality group.

2000 Mathematics Subject Classification: Primary 20C33, 20C15. Secondary 20D15, 20D20.

\section{Introduction}

Let $p$ be a fixed prime, $\mathbb{N}^{*}$ the set of positive integers, $q:=p^{k}$ with a fixed $k \in \mathbb{N}^{*}, \mathbb{F}_{q}$ the finite field with $q$ elements and $U_{n}(q)\left(n \in \mathbb{N}^{*}\right)$ the group of upper unitriangular $n \times n$-matrices (i.e. upper triangular matrices with 1 s on the main diagonal) with entries in $\mathbb{F}_{q}$. Then $U_{n}(q)$ is a Sylow $p$-subgroup of the general linear group $G L_{n}(q)$ of invertible $n \times n$-matrices over $\mathbb{F}_{q}$.

G. Higman [Hig60] formulated a longstanding conjecture: for a fixed $n$, the number of conjugacy classes of $U_{n}(q)$ is determined by a polynomial in $q$ with integral coefficients depending on $n$. G. Lehrer [Leh74] refined Higman's conjecture: the number of irreducible characters of $U_{n}(q)$ of degree $q^{c}(c \in \mathbb{N})$ is an integer polynomial in $q$. I. M. Isaacs [Isa95] proved that the degrees of complex irreducible characters of $\mathbb{F}_{q}$-algebra groups are powers of $q$. Then I. M. Isaacs [Isa07] gave a strengthened form of Lehrer's conjecture: the number of irreducible characters of degree $q^{c}$ is some polynomial in $(q-1)$ with non-negative integeral coefficients. A. Vera-López and J. M. Arregi [VLA03] proved Higman's conjecture for $n \leq 13$. Recently, I. Pak and A. Soffer [PS15] verified Higman's conjecture for $n \leq 16$.

It is natural to consider Higman's conjecture, Lehrer's conjecture and Isaacs' conjecture for the Sylow $p$-subgroups of other finite groups of Lie type. Let $G(q)$ be a finite group of Lie type, $U(q)$ a Sylow $p$-subgroup of $G(q), k(U(q))$ the number of conjugacy classes, \# $\operatorname{Irr}(U(q))$ the number of all complex irreducible characters, \# $\operatorname{Irr}\left(U(q), q^{c}\right)$ the number of complex irreducible characters of degree $q^{c}$, \#M(U(q)) the number of pairwise orthogonal irreducible constituents of the regular $U(q)$-module $\mathbb{C} U(q)$. S. M. Goodwin, P. Mosch and G. Röhrle [GR09, GMR14, GMR16] obtained an algorithm and calculated $k(U(q))$ for $U(q)$ of rank at most 8, except $E_{8}$.

For the Sylow $p$-subgroup $U(q)$ of the Chevalley group $D_{4}(q)$ of type $D_{4}$, F. Himstedt, T. Le and K. Magaard [HLM11] determined the complex irreducible characters and calculated $\# \operatorname{Irr}\left(U(q), q^{c}\right)$. M. Jedlitschky [Jed13, Appendix A.3] decomposed the regular module $\mathbb{C} U(q)$ with $p \neq 2$ into irreducible constituents, and obtained \# $\operatorname{Irr}\left(U(q), q^{c}\right)$ and $\# \operatorname{Irr}(U(q))$. S. M. Goodwin, T. Le and K. Magaard [GLM17] constructed the generic character table of $U(q)$. Higman's conjecture, Lehrer's conjecture and Isaacs' conjecture are true for $U(q)$. 
For the Sylow $p$-subgroup $U(q)(p>3)$ of the Chevalley group $G_{2}(q)$ of type $G_{2}$, F. Himstedt, T. Le and K. Magaard [HLM16] determined most irreducible characters of $U(q)$ by parameterizing midafis (minimal degree almost faithful irreducible characters).

S. M. Goodwin, T. Le, K. Magaard and A. Paolini [GLMP16] parameterized the irreducible characters of the Sylow $p$-subgroup $U(q)(p>2)$ of the Chevalley group $F_{4}(q)$ of type $F_{4}$.

Let $U$ be a Sylow $p$-subgroup of the Steinberg triality group ${ }^{3} D_{4}\left(q^{3}\right)$. T. Le [Le16] constructed and counted all ordinary irreducible characters of $U$, mainly using Clifford theory.

In this paper, let $p$ be a fixed odd prime, $q:=p^{k}$ a fixed power of $p$. In Section 2 , we recall the construction of a Sylow $p$-subgroup $U$ of the Steinberg triality group ${ }^{3} D_{4}\left(q^{3}\right)$. In Section 3, we establish the conjugacy classes and the explicit elements of the conjugacy classes of the Sylow $p$-subgroup $U$. Based on T. Le's work [Le16], we determine the specific constructions of all of the pairwise orthogonal complex irreducible characters of $U$ by Clifford's Theorem (see [CR81, Section 11]) in Section 4. Finally, we further calculate the values of the irreducible characters and obtain the character table of $U$ in Section 5,

If $p>2$, the classification of conjugacy classes of $U$ and the construction of irreducible character of $U$ are uniform. If $p=2$, the Lemma 3.6 does not hold, so the analysis is different. Some results still hold when their proofs do not use this lemma. For example, the conjugacy classes of $x=x\left(0,0,0, t_{4}, t_{5}, t_{6}\right) \in U\left(t_{4} \in \mathbb{F}_{q^{3}}, t_{5}, t_{6} \in \mathbb{F}_{q}\right)$ for $p=2$ are the same as these in Table 1 for $p>2$, but the conjugacy classes of $x_{3}\left(t_{3}^{*}\right) \in U\left(t_{3}^{*} \in \mathbb{F}_{q^{3}}^{*}\right)$ for $p=2$ and these for $p>2$ are different (see Remark 3.12). Similarly, the irreducible characters in the family $\mathfrak{F}_{4}$ of Proposition 4.12 do not hold when $p=2$. The construction of this case can be found in [Le16, 3.3]. The irreducible characters in the other cases for $p>2$ and for $p=2$ are the same (see Remark 4.14).

The main results in this paper are summarized in the following theorem:

1.1 Theorem. Let ${ }^{3} D_{4}\left(q^{3}\right)$ be the Steinberg triality group over $\mathbb{F}_{q^{3}}$ when $p>2$, and let $U$ be a Sylow p-subgroup of ${ }^{3} D_{4}\left(q^{3}\right)$. The conjugacy classes of $U$ are shown in Proposition 3.11. The explicit constructions of irreducible characters of $U$ are given in Proposition 4.12. In Proposition 5.1 the character table of $U$ is obtained.

Here we fix some notation: Let $K$ a field, $K^{*}$ the multiplicative group $K \backslash\{0\}$ of $K, K^{+}$the additive group of $K, \mathbb{F}_{q}$ the finite field with $q$ elements, $\mathbb{F}_{q^{3}}$ the finite field with $q^{3}$ elements, $\mathbb{C}$ the complex field, $\mathbb{Z}$ the set of all integers, $\mathbb{N}$ the set $\{0,1,2, \ldots\}$ of all non-negative integers, $\mathbb{N}^{*}$ the set $\{1,2, \ldots\}$ of all positive integers.

\section{Sylow $p$-subgroups $U$ of the Steinberg triality group ${ }^{3} D_{4}\left(q^{3}\right)$}

In this section, we recall the root system of $D_{4}$, the construction of a Sylow $p$-subgroup $U$ of the Steinberg triality group ${ }^{3} D_{4}\left(q^{3}\right)$, and the commutator relations of $U$. The main references are [Car72] and [Hum78].

Let $\mathcal{V}_{4}$ be a fixed Euclidean space with the usual orthonormal basis $\varepsilon_{1}, \varepsilon_{2}, \varepsilon_{3}, \varepsilon_{4}$, where the inner product ( , ) is the usual one. The set $\Phi_{D_{4}}=\left\{ \pm \varepsilon_{i} \pm \varepsilon_{j} \mid 1 \leq i<j \leq 4\right\}$ is a root system of type $D_{4}$, and $(r, r)=2$ for all $r \in \Phi_{D_{4}}$. The fundamental system of roots of the root system $\Phi_{D_{4}}$ is $\Delta_{D_{4}}=\left\{\varepsilon_{1}-\varepsilon_{2}, \varepsilon_{2}-\varepsilon_{3}, \varepsilon_{3}-\varepsilon_{4}, \varepsilon_{3}+\varepsilon_{4}\right\}$. The positive system (relative to $\Delta_{D_{4}}$ ) of roots of $\Phi_{D_{4}}$ is $\Phi_{D_{4}}^{+}:=\left\{\varepsilon_{i} \pm \varepsilon_{j} \mid 1 \leq i<j \leq 4\right\}$. Let $r_{1}:=\varepsilon_{1}-\varepsilon_{2}, r_{2}:=\varepsilon_{2}-\varepsilon_{3}, r_{3}:=\varepsilon_{3}-\varepsilon_{4}, r_{4}:=\varepsilon_{3}+\varepsilon_{4}$, then $\Delta_{D_{4}}=\left\{r_{1}, r_{2}, r_{3}, r_{4}\right\}$.

Let $r:=\sum_{i=1}^{4} x_{i} r_{i} \in \mathcal{V}_{4}, s:=\sum_{i=1}^{4} y_{i} r_{i} \in \mathcal{V}_{4}$, then we write $r \prec s$, if $\sum_{i=1}^{4} x_{i}<\sum_{i=1}^{4} y_{i}$, or if $\sum_{i=1}^{4} x_{i}=\sum_{i=1}^{4} y_{i}$ and the first non-zero coefficient $x_{i}-y_{i}$ is positive. If $r=\sum_{i=1}^{4} x_{i} r_{i} \in \Phi_{D_{4}}$, the height of $r$ (relative to $\Delta_{D_{4}}$ ) is denoted by ht $(r):=\sum_{i=1}^{4} x_{i}$. Let $r_{5}:=r_{1}+r_{2}, r_{6}:=$ $r_{2}+r_{3}, r_{7}:=r_{2}+r_{4}, r_{8}:=r_{1}+r_{2}+r_{3}, r_{9}:=r_{1}+r_{2}+r_{4}, r_{10}:=r_{2}+r_{3}+r_{4}, r_{11}:=$ 
$r_{1}+r_{2}+r_{3}+r_{4}, r_{12}:=r_{1}+2 r_{2}+r_{3}+r_{4}$, then $\Phi_{D_{4}}^{+}=\left\{r_{i} \mid i=1,2, \ldots, 12\right\}$. We get the total order of $\Phi_{D_{4}}^{+}$as follows:

$$
0 \prec r_{1} \prec r_{2} \prec r_{3} \prec r_{4} \prec r_{5} \prec r_{6} \prec r_{7} \prec r_{8} \prec r_{9} \prec r_{10} \prec r_{11} \prec r_{12} .
$$

Let $\mathcal{L}$ be a complex simple Lie algebra of type $D_{4}$ with a Cartan subalgebra $\mathcal{H}$, a Cartan decomposition $\mathcal{L}=\mathcal{H} \oplus \sum_{r \in \Phi_{D_{4}}} \mathcal{L}_{r}$, and a Chevalley basis $\left\{h_{r} \mid r \in \Delta_{D_{4}}\right\} \cup\left\{e_{r} \mid r \in \Phi_{D_{4}}\right\}$, where $e_{r} \in \mathcal{L}_{r}$ for all $r \in \Phi_{D_{4}}$. Then $\left[e_{r}, e_{s}\right]=N_{r, s} e_{r+s}$ for all $r, s \in \Phi_{D_{4}}$, where $N_{r, s}= \pm\left(n_{r, s}+1\right)$ and $n_{r, s}$ is the greatest integer for which $s-n_{r, s} r \in \Phi_{D_{4}}$.

We choose the signs of the structure constants $N_{r, s}$ for extra special pairs $(r, s)$ (see [Car72, Page 58]) as follows:

$$
\begin{array}{lll}
-N_{r_{1}, r_{2}}=N_{r_{2}, r_{3}}=N_{r_{2}, r_{4}}=1, & & N_{r_{1}, r_{2}+r_{3}+r_{4}}=1, \\
N_{r_{1}, r_{2}+r_{3}}=N_{r_{1}, r_{2}+r_{4}}=N_{r_{2}, r_{3}+r_{4}}=1, & & N_{r_{2}, r_{1}+r_{2}+r_{3}+r_{4}}=1 .
\end{array}
$$

Then the structure constants of the other special pairs (see [Car72, Page 58]) of $r_{i} \in \Phi_{D_{4}}^{+} \backslash \Delta_{D_{4}}$ are 1.

Let $x \in \mathcal{L}$. We define a linear map by ad $x: \mathcal{L} \rightarrow \mathcal{L}: y \mapsto[x, y]:=x y-y x$. Let $x_{r}(t):=$ $\exp \left(t\right.$ ade $\left.e_{r}\right)$ for all $r \in \Phi_{D_{4}}, t \in \mathbb{C}$. Let $K$ be a field, then the Chevalley group of $\mathcal{L}$ over $K$ is defined by $D_{4}(K):=\left\langle x_{r}(t) \mid r \in \Phi_{D_{4}}, t \in K\right\rangle$. The Chevalley group of $\mathcal{L}$ over $\mathbb{F}_{q}$ is also denoted by $D_{4}(q)$. Let $r \in \Phi_{D_{4}}$, then the root subgroup is denoted by $X_{r}:=\left\langle x_{r}(t) \mid t \in K\right\rangle$. The subgroup $\left\langle x_{r}(t) \mid r \in \Phi_{D_{4}}^{+}, t \in \mathbb{F}_{q}\right\rangle$ is a Sylow $p$-subgroup of $D_{4}(q)$.

Let $\sigma$ be a non-trivial symmetry of the Dynkin diagram of $\mathcal{L}_{D_{4}}$ sending $r_{1}$ to $r_{3}, r_{3}$ to $r_{4}, r_{4}$ to $r_{1}$, and fixing $r_{2}, \rho$ be a linear transformation of $\mathcal{V}_{4}$ into itself arising from $\sigma$, then $\rho\left(\Phi_{D_{4}}\right)=\Phi_{D_{4}}$. The Chevalley group $D_{4}\left(q^{3}\right)$ has a field automorphism $F_{q}: \mathbb{F}_{q^{3}} \rightarrow \mathbb{F}_{q^{3}}: t \mapsto t^{q}$ sending $x_{r}(t)$ to $x_{r}\left(t^{q}\right)$, and a graph automorphism $\rho$ sending $x_{r}(t)$ to $x_{\rho(r)}(t)\left(r \in \Phi_{D_{4}}\right)$. Let $F:=\rho F_{q}=F_{q} \rho$. For a subgroup $X$ of $D_{4}\left(q^{3}\right)$, set $X^{F}:=\{x \in X \mid F(x)=x\}$. Then we get $D_{4}\left(q^{3}\right)^{F}={ }^{3} D_{4}\left(q^{3}\right)$. Set $r^{1}:=\frac{1}{3}\left(r+\rho(r)+\rho^{2}(r)\right)$ for all $r \in \Phi_{D_{4}}$. Let $\alpha:=\frac{1}{3}\left(r_{1}+r_{3}+r_{4}\right)$ and $\beta:=r_{2}$, then $\Delta_{G_{2}}:=\{\alpha, \beta\}$ ( $\alpha$ is short) is a fundamental system of type $G_{2}$, and the root system of type $G_{2}$ is

$$
\Phi_{G_{2}}=\left\{r^{1} \mid r \in \Phi_{D_{4}}\right\}=\{ \pm \alpha, \pm \beta, \pm(\alpha+\beta), \pm(2 \alpha+\beta), \pm(3 \alpha+\beta), \pm(3 \alpha+2 \beta)\} .
$$

We obtain the following elements of ${ }^{3} D_{4}\left(q^{3}\right)$. Let $r \in \Phi_{D_{4}}$ and $t \in \mathbb{F}_{q^{3}}$, we set

$$
x_{r^{1}}(t):=\left\{\begin{array}{ll}
x_{r}(t) & \text { if } \rho(r)=r, t^{q}=t \\
x_{r}(t) x_{\rho(r)}\left(t^{q}\right) x_{\rho^{2}(r)}\left(t^{q^{2}}\right) & \text { if } \rho(r) \neq r, t^{q^{3}}=t
\end{array} .\right.
$$

The root subgroups of ${ }^{3} D_{4}\left(q^{3}\right)$ are $X_{r^{1}}:=\left\{x_{r^{1}}(t) \mid t \in \mathbb{F}_{q^{3}}\right\}$ for all $r \in \Phi_{D_{4}}$.

We write

$$
\begin{array}{ll}
x_{1}(t):=x_{r_{1}^{1}}(t)=x_{r_{1}}(t) x_{r_{3}}\left(t^{q}\right) x_{r_{4}}\left(t^{q^{2}}\right) & \text { for all } t \in \mathbb{F}_{q^{3}}, \\
x_{2}(t):=x_{r_{2}^{1}}(t)=x_{r_{2}}(t) & \text { for all } t \in \mathbb{F}_{q}, \\
x_{3}(t):=x_{r_{5}^{1}}(t)=x_{r_{5}}(t) x_{r_{6}}\left(t^{q}\right) x_{r_{7}}\left(t^{q^{2}}\right) & \text { for all } t \in \mathbb{F}_{q^{3}}, \\
x_{4}(t):=x_{r_{8}^{1}}(t)=x_{r_{8}}(t) x_{r_{10}}\left(t^{q}\right) x_{r_{9}}\left(t^{q^{2}}\right) & \text { for all } t \in \mathbb{F}_{q^{3}}, \\
x_{5}(t):=x_{r_{11}^{1}}(t)=x_{r_{11}}(t) & \text { for all } t \in \mathbb{F}_{q}, \\
x_{6}(t):=x_{r_{12}^{1}}(t)=x_{r_{12}}(t) & \text { for all } t \in \mathbb{F}_{q} .
\end{array}
$$

Set $X_{i}:=\left\{x_{i}(t) \mid t \in \mathbb{F}_{q^{3}}\right\}(i=1,3,4)$ and $X_{i}:=\left\{x_{i}(t) \mid t \in \mathbb{F}_{q}\right\}(i=2,5,6)$. Note that the root subgroups are denoted by $Y_{i}$ in [Le16], and $Y_{1}:=X_{2}, Y_{2}:=X_{1}$ and $Y_{i}:=X_{i}$ for $i=3,4,5,6$. We obtain a Sylow $p$-subgroup $U$ of ${ }^{3} D_{4}\left(q^{3}\right)$ :

$$
U:=\left\{x_{2}\left(t_{2}\right) x_{1}\left(t_{1}\right) x_{3}\left(t_{3}\right) x_{4}\left(t_{4}\right) x_{5}\left(t_{5}\right) x_{6}\left(t_{6}\right) \mid t_{1}, t_{3}, t_{4} \in \mathbb{F}_{q^{3}}, t_{2}, t_{5}, t_{6} \in \mathbb{F}_{q}\right\},
$$


where the product can be taken in an arbitrary, but fixed, order.

Let $t_{1}, t_{3}, t_{4} \in \mathbb{F}_{q^{3}}, t_{2}, t_{5}, t_{6} \in \mathbb{F}_{q}$ and define the commutator

$$
\left[x_{i}\left(t_{i}\right), x_{j}\left(t_{j}\right)\right]:=x_{i}\left(t_{i}\right)^{-1} x_{j}\left(t_{j}\right)^{-1} x_{i}\left(t_{i}\right) x_{j}\left(t_{j}\right) .
$$

Then the non-trivial commutators of $U$ are determined as follows:

$$
\begin{aligned}
& {\left[x_{1}\left(t_{1}\right), x_{2}\left(t_{2}\right)\right]=x_{3}\left(-t_{2} t_{1}\right) x_{4}\left(t_{2} t_{1}^{q+1}\right) x_{5}\left(-t_{2} t_{1}^{q^{2}+q+1}\right) x_{6}\left(2 t_{2}^{2} t_{1}^{q^{2}+q+1}\right),} \\
& {\left[x_{1}\left(t_{1}\right), x_{3}\left(t_{3}\right)\right]=x_{4}\left(t_{1} t_{3}^{q}+t_{1}^{q} t_{3}\right) x_{5}\left(-t_{1}^{q+1} t_{3}^{q^{2}}-t_{1}^{q^{2}+q} t_{3}-t_{1}^{q^{2}+1} t_{3}^{q}\right) x_{6}\left(-t_{1} t_{3}^{q^{2}+q}-t_{1}^{q} t_{3}^{q^{2}+1}-t_{1}^{q^{2}} t_{3}^{q+1}\right),} \\
& {\left[x_{1}\left(t_{1}\right), x_{4}\left(t_{4}\right)\right]=x_{5}\left(t_{1} t_{4}^{q}+t_{1}^{q} t_{4}^{q^{2}}+t_{1}^{q^{2}} t_{4}\right),} \\
& {\left[x_{3}\left(t_{3}\right), x_{4}\left(t_{4}\right)\right]=x_{6}\left(t_{3} t_{4}^{q}+t_{3}^{q} t_{4}^{q^{2}}+t_{3}^{q^{2}} t_{4}\right),} \\
& {\left[x_{2}\left(t_{2}\right), x_{5}\left(t_{5}\right)\right]=x_{6}\left(t_{2} t_{5}\right) .}
\end{aligned}
$$

From now on, we set

$$
x\left(t_{1}, t_{2}, t_{3}, t_{4}, t_{5}, t_{6}\right):=x_{2}\left(t_{2}\right) x_{1}\left(t_{1}\right) x_{3}\left(t_{3}\right) x_{4}\left(t_{4}\right) x_{5}\left(t_{5}\right) x_{6}\left(t_{6}\right) \in U .
$$

\section{Conjugacy classes}

In this section, let $p$ be a fixed odd prime, we determine the conjugacy classes of the Sylow $p$-subgroup $U$ of ${ }^{3} D_{4}\left(q^{3}\right)$.

3.1 Notation. Let $x, u \in U$, then the conjugate of $x$ by $u$ is defined by ${ }^{u} x:=u x u^{-1}$, and the conjugacy class of $x$ is the $\operatorname{set}^{U} x:=\left\{v x v^{-1} \mid v \in U\right\}$.

3.2 Lemma. Let $\phi_{0}: \mathbb{F}_{q^{3}} \rightarrow \mathbb{F}_{q}: t \mapsto t+t^{q}+t^{q^{2}}$, then $\phi_{0}$ is an $\mathbb{F}_{q^{-}}$epimorphism and $\left|\operatorname{ker} \phi_{0}\right|=q^{2}$.

Proof. Let $t, s \in \mathbb{F}_{q^{3}}$ and $k \in \mathbb{F}_{q}$, then $\phi_{0}(t+s)=(t+s)+(t+s)^{q}+(t+s)^{q^{2}}=\left(t+t^{q}+t^{q^{2}}\right)+$ $\left(s+s^{q}+s^{q^{2}}\right)=\phi_{0}(t)+\phi_{0}(s)$, and $\phi_{0}(k t)=(k t)+(k t)^{q}+(k t)^{q^{2}}=k\left(t+t^{q}+t^{q^{2}}\right)=k \phi_{0}(t)$. Thus $\phi_{0}$ is an $\mathbb{F}_{q}$-homomorphism. Observe that ker $\phi_{0}=\left\{t \in \mathbb{F}_{q^{3}} \mid t+t^{q}+t^{q^{2}}=0\right\}$. The degree of $t+t^{q}+t^{q^{2}}$ is $q^{2}$, so $\left|\operatorname{ker} \phi_{0}\right| \leq q^{2}$. On the other hand, $\left|\operatorname{im} \phi_{0}\right| \leq q$ since im $\phi_{0} \subseteq \mathbb{F}_{q}$. Then $\left|\operatorname{ker} \phi_{0}\right|=\frac{\left|\mathbb{F}_{q^{3}}\right|}{\left|\operatorname{im} \phi_{0}\right|} \geq q^{2}$ since $\mathbb{F}_{q^{3}} / \operatorname{ker} \phi_{0} \cong \operatorname{im} \phi_{0}$. Thus $\left|\operatorname{ker} \phi_{0}\right|=q^{2}$ and $\operatorname{im} \phi_{0}=\mathbb{F}_{q}$. Therefore, $\phi_{0}$ is an $\mathbb{F}_{q}$-epimorphism.

3.3 Proposition. There exists an element $\eta \in \mathbb{F}_{q^{3}} \backslash \mathbb{F}_{q}$ (i.e. $\eta \in \mathbb{F}_{q^{3}}$ but $\eta \notin \mathbb{F}_{q}$ ) such that $\eta^{q^{2}}+$ $\eta^{q}+\eta=1$.

Proof. By 3.2, we obtain $\#\left\{t \in \mathbb{F}_{q^{3}} \mid t^{q^{2}}+t^{q}+t=1\right\}=q^{2}$. But $\left|\mathbb{F}_{q}\right|=q$, so there are at least $q^{2}-q(>1)$ elements of $\mathbb{F}_{q^{3}}$ which satisfy the equation $t^{q^{2}}+t^{q}+t=1$. Then the claim is proved.

From now on, we fix an element $\eta \in \mathbb{F}_{q^{3}} \backslash \mathbb{F}_{q}$ such that $\eta^{q^{2}}+\eta^{q}+\eta=1$.

3.4 Corollary. $1+\eta^{1-q^{2}} \neq 0$.

Proof. Suppose that $1+\eta^{1-q^{2}}=0$. Multiply $\eta^{q^{2}}(\neq 0)$ to both sides of the formula, then $\eta^{q^{2}}+\eta=0$, by $3.3 \eta^{q}=1$, so $\eta=1 \in \mathbb{F}_{q}$. This is a contradiction to that $\eta \in \mathbb{F}_{q^{3}} \backslash \mathbb{F}_{q}$.

3.5 Notation/Lemma. The map $\pi_{q}: \mathbb{F}_{q^{3}} \rightarrow \mathbb{F}_{q}: x \mapsto \phi_{0}(\eta x)=(\eta x)^{q^{2}}+(\eta x)^{q}+\eta x$ is an $\mathbb{F}_{q^{-}}$epimorphism and $\mathbb{F}_{q^{3}}=\operatorname{ker} \pi_{q} \oplus \mathbb{F}_{q}$. In particular, $\left.\pi_{q}\right|_{\mathbb{F}_{q}}=\mathrm{id}_{\mathbb{F}_{q}}$ and $\pi_{q}^{2}=\pi_{q}$.

3.6 Lemma. Let $p>2$ and $u \in \mathbb{F}_{q^{3}}^{*}$, then the map $\zeta_{u}: \mathbb{F}_{q^{3}} \rightarrow \mathbb{F}_{q^{3}}: t \mapsto u t^{q^{2}}+u^{q} t^{q}$ is an $\mathbb{F}_{q}$-automorphism. 
Proof. Since taking the $q$-th power is an $\mathbb{F}_{q}$-homomorphism, $\zeta_{u}$ is an $\mathbb{F}_{q}$-homomorphism. Let $t \in \operatorname{ker} \zeta_{u}$, then $0=\zeta_{u}(t)=u t^{q^{2}}+u^{q} t^{q}=\left(u^{1-q} t^{q^{2}-q}+1\right) u^{q} t^{q}=\left(\left(u^{-1} t^{q}\right)^{q-1}+1\right) u^{q} t^{q}$. Suppose that $t \neq 0$, then $\left(u^{-1} t^{q}\right)^{q-1}=-1$ and $\left(u^{-1} t^{q}\right)^{2(q-1)}=1$. Thus the order of $\left(u^{-1} t^{q}\right)^{q-1}$ is 2, i.e. $\left|\left(u^{-1} t^{q}\right)^{q-1}\right|=2$. On the other hand, since $u^{-1} t^{q} \in \mathbb{F}_{q^{3}}^{*}$, the order $\left|u^{-1} t^{q}\right|$ divides $\left(q^{3}-1\right)=(q-1)\left(q^{2}+q+1\right)$, so $\left|\left(u^{-1} t^{q}\right)^{q-1}\right|$ divides $\left(q^{2}+q+1\right)$. Since $q^{2}+q+1$ is odd, we get $\left|\left(u^{-1} t^{q}\right)^{q-1}\right| \neq 2$. This is a contradiction, so $\operatorname{ker} \zeta_{u}=\{0\}$. Thus $\mathbb{F}_{q^{3}} \cong \operatorname{im} \zeta_{u} \subseteq \mathbb{F}_{q^{3}}$, then $\operatorname{im} \zeta_{u}=\mathbb{F}_{q^{3}}$. Therefore, $\zeta_{u}$ is an $\mathbb{F}_{q^{-}}$automorphism.

3.7 Corollary. Let $p>2$, the map $\mathbb{F}_{q^{3}} \rightarrow \mathbb{F}_{q^{3}}: t \mapsto t+t^{q}$ is an $\mathbb{F}_{q^{-}}$automorphism.

We obtain the following conjugate elements from the commutator relations of $U$.

3.8 Lemma. Let $u:=x\left(r_{1}, r_{2}, r_{3}, r_{4}, r_{5}, r_{6}\right) \in U, x_{i}\left(t_{i}\right) \in U$ and $\bar{t}_{3} \in \mathbb{F}_{q^{3}}$, then

$$
\begin{aligned}
{ }^{u} x_{6}\left(t_{6}\right)= & x_{6}\left(t_{6}\right), \\
{ }^{u} x_{5}\left(t_{5}\right)= & x_{5}\left(t_{5}\right) \cdot x_{6}\left(r_{2} t_{5}\right), \\
{ }^{u} x_{4}\left(t_{4}\right)= & x_{4}\left(t_{4}\right) \cdot x_{5}\left(\phi_{0}\left(r_{1} t_{4}^{q}\right)\right) \cdot x_{6}\left(\phi_{0}\left(r_{1} r_{2} t_{4}^{q}\right)+\phi_{0}\left(r_{3} t_{4}^{q}\right)\right), \\
{ }^{u} x_{3}\left(t_{3}\right)= & x_{3}\left(t_{3}\right) \cdot x_{4}\left(r_{1} t_{3}^{q}+r_{1}^{q} t_{3}\right) \cdot x_{5}\left(\phi_{0}\left(r_{1}^{q^{2}+q} t_{3}\right)\right) \\
& \cdot x_{6}\left(\phi_{0}\left(r_{1}^{q^{2}+q} r_{2} t_{3}\right)+\phi_{0}\left(-r_{1} t_{3}^{q^{2}+q}\right)+\phi_{0}\left(-t_{3} r_{4}^{q}\right)\right), \\
{ }^{u} x_{2}\left(t_{2}\right)= & x_{2}\left(t_{2}\right) \cdot x_{3}\left(-r_{1} t_{2}\right) \cdot x_{4}\left(-t_{2} r_{1}^{q+1}\right) \cdot x_{5}\left(-t_{2} r_{1}^{q^{2}+q+1}\right) \\
& \cdot x_{6}\left(-t_{2} r_{5}-t_{2}^{2} r_{1}^{q^{2}+q+1}-t_{2} r_{1}^{q^{2}+q+1} r_{2}\right), \\
{ }^{u} x_{1}\left(t_{1}\right)= & x_{1}\left(t_{1}\right) \cdot x_{3}\left(r_{2} t_{1}\right) \cdot x_{4}\left(-r_{2} t_{1}^{q+1}-t_{1} r_{3}^{q}-t_{1}^{q} r_{3}\right) \\
& \cdot x_{5}\left(r_{2} t_{1}^{q^{2}+q+1}+\phi_{0}\left(r_{1}^{q^{2}}\left(-r_{3} t_{1}^{q}-r_{3}^{q} t_{1}\right)\right)+\phi_{0}\left(r_{3}^{q^{2}} t_{1}^{q+1}\right)+\phi_{0}\left(-t_{1} r_{4}^{q}\right)\right) \\
& \cdot x_{6}\left(2 r_{2}^{2} t_{1}^{q^{2}+q+1}+\phi_{0}\left(r_{1}^{q^{2}} r_{2}\left(-r_{3} t_{1}^{q}-r_{3}^{q} t_{1}\right)\right)+\phi_{0}\left(r_{2} r_{3}^{q^{2}} t_{1}^{q+1}\right)\right. \\
& \left.\quad+\phi_{0}\left(-r_{2} r_{4}^{q} t_{1}\right)+\phi_{0}\left(-t_{1} r_{3}^{q^{2}+q}\right)\right),
\end{aligned}
$$

and

$$
\begin{gathered}
u\left(x_{3}\left(t_{3}\right) x_{5}\left(t_{5}\right)\right)=x_{3}\left(t_{3}\right) \cdot x_{4}\left(r_{1} t_{3}^{q}+r_{1}^{q} t_{3}\right) \cdot x_{5}\left(t_{5}+\phi_{0}\left(r_{1}^{q^{2}+q} t_{3}\right)\right) \\
\cdot x_{6}\left(r_{2} t_{5}+\phi_{0}\left(r_{1}^{q^{2}+q} r_{2} t_{3}\right)+\phi_{0}\left(-r_{1} t_{3}^{q^{2}+q}\right)+\phi_{0}\left(-t_{3} r_{4}^{q}\right)\right), \\
\begin{aligned}
& u\left(x_{2}\left(t_{2}\right) x_{4}\left(t_{4}\right) x_{5}\left(t_{5}\right)\right)= x_{2}\left(t_{2}\right) \cdot x_{3}\left(-r_{1} t_{2}\right) \cdot x_{4}\left(t_{4}-t_{2} r_{1}^{q+1}\right) \cdot x_{5}\left(t_{5}-t_{2} r_{1}^{q^{2}+q+1}+\phi_{0}\left(r_{1} t_{4}^{q}\right)\right) \\
& x_{6}\left(-t_{2} r_{5}-t_{2}^{2} r_{1}^{q^{2}+q+1}-t_{2} r_{1}^{q^{2}+q+1} r_{2}+\phi_{0}\left(r_{1} r_{2} t_{4}^{q}\right)+\phi_{0}\left(r_{3} t_{4}^{q}\right)+r_{2} t_{5}\right), \\
&=x_{1}\left(t_{1}\right) \cdot x_{3}\left(r_{2} t_{1}+\bar{t}_{3}\right) \cdot x_{4}\left(-r_{2} t_{1}^{q+1}-t_{1} r_{3}^{q}-t_{1}^{q} r_{3}+r_{1} \bar{t}_{3}^{q}+r_{1}^{q} \bar{t}_{3}\right) \\
& \cdot x_{5}\left(r_{2} t_{1}^{q^{2}+q+1}+\phi_{0}\left(r_{1}^{q^{2}}\left(-r_{3} t_{1}^{q}-r_{3}^{q} t_{1}\right)\right)+\phi_{0}\left(r_{3}^{q^{2}} t_{1}^{q+1}\right)+\phi_{0}\left(-t_{1} r_{4}^{q}\right)+\phi_{0}\left(r_{1}^{q^{2}+q} \bar{t}_{3}\right)\right) \\
& \cdot x_{6}\left(2 r_{2}^{2} t_{1}^{q^{2}+q+1}+\phi_{0}\left(r_{1}^{q^{2}} r_{2}\left(-r_{3} t_{1}^{q}-r_{3}^{q} t_{1}\right)\right)+\phi_{0}\left(r_{2} r_{3}^{q^{2}} t_{1}^{q+1}\right)+\phi_{0}\left(-r_{2} r_{4}^{q} t_{1}\right)+\phi_{0}\left(-t_{1} r_{3}^{q^{2}+q}\right)\right. \\
&\left.+\phi_{0}\left(r_{1}^{q^{2}+q} r_{2} \bar{t}_{3}\right)+\phi_{0}\left(-r_{1} \bar{t}_{3}^{q^{2}+q}\right)+\phi_{0}\left(-\bar{t}_{3} r_{4}^{q}\right)+\phi_{0}\left(\bar{t}_{3}^{q^{2}}\left(r_{2} t_{1}^{q+1}+t_{1} r_{3}^{q}+t_{1}^{q} r_{3}\right)\right)\right),
\end{aligned}
\end{gathered}
$$




$$
\begin{aligned}
& u\left(x_{2}\left(t_{2}\right) x_{1}\left(t_{1}\right)\right) \\
= & x_{2}\left(t_{2}\right) x_{1}\left(t_{1}\right) \cdot x_{3}\left(r_{2} t_{1}-r_{1} t_{2}\right) \cdot x_{4}\left(-r_{2} t_{1}^{q+1}-t_{1} r_{3}^{q}-t_{1}^{q} r_{3}-t_{2} r_{1}^{q+1}+t_{1} t_{2} r_{1}^{q}+t_{1}^{q} t_{2} r_{1}\right) \\
& \cdot x_{5}\left(r_{2} t_{1}^{q^{2}+q+1}+\phi_{0}\left(r_{1}^{q^{2}}\left(-r_{3} t_{1}^{q}-r_{3}^{q} t_{1}\right)\right)+\phi_{0}\left(r_{3}^{q^{2}} t_{1}^{q+1}\right)+\phi_{0}\left(-t_{1} r_{4}^{q}\right)\right. \\
& \left.\quad-t_{2} r_{1}^{q^{2}+q+1}+\phi_{0}\left(-r_{1} t_{1}^{q^{2}+q} t_{2}\right)+\phi_{0}\left(t_{1}^{q^{2}} t_{2} r_{1}^{q+1}\right)\right) \\
& \cdot x_{6}\left(2 r_{2}^{2} t_{1}^{q^{2}+q+1}+\phi_{0}\left(r_{1}^{q^{2}} r_{2}\left(-r_{3} t_{1}^{q}-r_{3}^{q} t_{1}\right)\right)+\phi_{0}\left(r_{2} r_{3}^{q^{2}} t_{1}^{q+1}\right)+\phi_{0}\left(-r_{2} r_{4}^{q} t_{1}\right)+\phi_{0}\left(-t_{1} r_{3}^{q^{2}+q}\right)\right. \\
& \quad-t_{2} r_{5}-t_{2}^{2} r_{1}^{q^{2}+q+1}-t_{2} r_{1}^{q^{2}+q+1} r_{2}+\phi_{0}\left(-2 r_{1} r_{2} t_{1}^{q^{2}+q} t_{2}\right)+\phi_{0}\left(t_{1}^{q^{2}} t_{2} r_{1}^{q+1} r_{2}\right) \\
& \left.+\phi_{0}\left(r_{1}^{q^{2}+q_{1}} t_{1} t_{2}^{2}\right)\right) .
\end{aligned}
$$

Before we get the conjugacy classes of $U$, we need the following lemma.

3.9 Lemma. Let $u \in \mathbb{F}_{q^{3}} \backslash \mathbb{F}_{q}$, ker $\phi_{0}$ be the kernel of $\phi_{0}, S:=u$ ker $\phi_{0}$ and

$$
\left.\phi_{0}\right|_{S}: u \operatorname{ker} \phi_{0} \rightarrow \mathbb{F}_{q}: t \mapsto t^{q^{2}}+t^{q}+t .
$$

Then $\left.\phi_{0}\right|_{S}$ is an $\mathbb{F}_{q}$-epimorphism.

Proof. The kernel ker $\phi_{0}$ is an $\mathbb{F}_{q^{-}}$-vector subspace of $\mathbb{F}_{q^{3}}$ and $u \in \mathbb{F}_{q^{3}}$, so $u$ ker $\phi_{0}$ is an $\mathbb{F}_{q^{-}}$-vector subspace of $\mathbb{F}_{q^{3}}$. Thus $\left.\phi_{0}\right|_{S}$ is an $\mathbb{F}_{q}$-homomorphism by 3.2. Let $t_{0} \in \operatorname{ker} \phi_{0}$ and $u t_{0} \in \operatorname{ker}\left(\left.\phi_{0}\right|_{S}\right)$, then $t_{0}^{q^{2}}+t_{0}^{q}+t_{0}=0$ and $u^{q^{2}} t_{0}^{q^{2}}+u^{q} t_{0}^{q}+u t_{0}=0$, so $u^{q^{2}} t_{0}^{q^{2}}+u^{q^{2}} t_{0}^{q}+u^{q^{2}} t_{0}=0$ since $u^{q^{2}} \neq 0$. Then $\left(u^{q}-u^{q^{2}}\right) t_{0}^{q}+\left(u-u^{q^{2}}\right) t_{0}=0$. Since $u \notin \mathbb{F}_{q}, u^{q}-u^{q^{2}} \neq 0$. Then $\left(u^{q}-u^{q^{2}}\right)^{-1} t_{0}=\left(u^{q}-u^{q^{2}}\right)^{-q} t_{0}^{q}$, so $\left(u^{q}-u^{q^{2}}\right)^{-1} t_{0} \in \mathbb{F}_{q}$ and $t_{0} \in\left(u^{q}-u^{q^{2}}\right) \mathbb{F}_{q}$. Thus $\left|\operatorname{ker}\left(\left.\phi_{0}\right|_{S}\right)\right| \leq\left|u\left(u^{q}-u^{q^{2}}\right) \mathbb{F}_{q}\right|=q$, then $\left|\operatorname{im}\left(\left.\phi_{0}\right|_{S}\right)\right| \geq \frac{|S|}{\left|\operatorname{ker}\left(\left.\phi_{0}\right|_{S}\right)\right|} \stackrel{3.2}{=} \frac{q^{2}}{q}=q$. We have $\left|\operatorname{im}\left(\left.\phi_{0}\right|_{S}\right)\right|=q$ since $\left|\operatorname{im}\left(\left.\phi_{0}\right|_{S}\right)\right| \leq\left|\mathbb{F}_{q}\right|=q$. Therefore, $\left.\phi_{0}\right|_{S}$ is an $\mathbb{F}_{q}$-epimorphism.

3.10 Notation. Let $a^{*} \in \mathbb{F}_{q^{3}}^{*}=\mathbb{F}_{q^{3}} \backslash\{0\}$, then denote by $T^{a^{*}}$ a complete set of coset representatives (i.e. a transversal) of $\left(a^{*} \mathbb{F}_{q}^{+}\right)$in $\mathbb{F}_{q^{3}}^{+}$. Thus $\left|T^{a^{*}}\right|=q^{2}$. If $\bar{t}_{0} \in T^{a^{*}}$ and $\bar{t}_{0} \in a^{*} \mathbb{F}_{q}^{+}$, we set $\bar{t}_{0}=0$.

3.11 Proposition (Conjugacy classes of $U$ ). Let $p>2$, the conjugacy classes of the Sylow $p$ subgroup $U$ of ${ }^{3} D_{4}\left(q^{3}\right)$ are listed in Table 1

Proof. We prove the hard cases of the proposition. Let $0 \neq t_{1} \in \mathbb{F}_{q^{*}}^{*}, \bar{t}_{3} \in \mathbb{F}_{q^{3}}, t_{6} \in \mathbb{F}_{q}$, $u:=x\left(r_{1}, r_{2}, r_{3}, r_{4}, r_{5}, r_{6}\right) \in U$ and $x\left(a_{1}, a_{2}, a_{3}, a_{4}, a_{5}, a_{6}\right):={ }^{u}\left(x_{1}\left(t_{1}\right) x_{3}\left(\bar{t}_{3}\right) x_{6}\left(t_{6}\right)\right)$.

(1) Let $\bar{t}_{3} \in t_{1} \mathbb{F}_{q}^{+}$, then there exists $s \in \mathbb{F}_{q}^{+}$such that $\bar{t}_{3}=s t_{1}$. By 3.8 ,

$$
\begin{aligned}
a_{1}= & t_{1}, a_{2}=0, a_{3}=r_{2} t_{1}+\bar{t}_{3}=\left(r_{2}+s\right) t_{1}, a_{4}=-r_{2} t_{1}^{q+1}-\left(r_{3}-s r_{1}\right) t_{1}^{q}-\left(r_{3}-s r_{1}\right)^{q} t_{1}, \\
a_{5}= & r_{2} t_{1}^{q^{2}+q+1}+\phi_{0}\left(r_{1}^{q^{2}}\left(-r_{3} t_{1}^{q}-r_{3}^{q} t_{1}\right)\right)+\phi_{0}\left(r_{3}^{q^{2}} t_{1}^{q+1}\right)+\phi_{0}\left(-t_{1} r_{4}^{q}\right)+\phi_{0}\left(s r_{1}^{q^{2}+q} t_{1}\right), \\
a_{6}= & t_{6}+r_{2}^{2} t_{1}^{q^{2}+q+1}+r_{2} a_{5}+\phi_{0}\left(-t_{1} r_{3}^{q^{2}+q}\right)+\phi_{0}\left(-s^{2} r_{1} t_{1}^{q^{2}+q}\right) \\
& +\phi_{0}\left(-s t_{1} r_{4}^{q}\right)+\phi_{0}\left(s t_{1}^{q^{2}}\left(r_{2} t_{1}^{q+1}+t_{1} r_{3}^{q}+t_{1}^{q} r_{3}\right)\right) \\
= & t_{6}+r_{2}^{2} t_{1}^{q^{2}+q+1}+\left(r_{2}+s\right) a_{5}+2 s r_{2} t_{1}^{q^{2}+q+1} \\
& +\phi_{0}\left(s\left(r_{3}-s r_{1}\right) t_{1}^{q^{2}+q}\right)-\phi_{0}\left(\left(r_{3}-s r_{1}\right)^{q^{2}+q} t_{1}\right) .
\end{aligned}
$$

Let $a_{3}, a_{4}$ and $a_{5}$ be fixed, then $a_{6}$ is determined uniquely by 3.2 and 3.6 . Hence we get the conjugacy class of $x_{1}\left(t_{1}\right) x_{6}\left(t_{6}\right)$.

$$
U\left(x_{1}\left(t_{1}\right) x_{6}\left(t_{6}\right)\right)=\left\{x\left(t_{1}, 0, s_{2} t_{1}, s_{4}, s_{5}, \hat{s}_{6}\right) \mid s_{4} \in \mathbb{F}_{q^{3}}, s_{2}, s_{5} \in \mathbb{F}_{q}\right\} .
$$


Table 1: Conjugacy classes of $U$ for $p>2$

\begin{tabular}{|c|c|c|}
\hline Representatives $x \in U$ & Conjugacy Classes ${ }^{U} x$ & $\left|{ }^{U} x\right|$ \\
\hline$I_{8}$ & $x(0,0,0,0,0,0)$ & 1 \\
\hline$x_{6}\left(t_{6}^{*}\right), t_{6}^{*} \in \mathbb{F}_{q}^{*}$ & $x\left(0,0,0,0,0, t_{6}^{*}\right)$ & 1 \\
\hline$x_{5}\left(t_{5}^{*}\right), t_{5}^{*} \in \mathbb{F}_{q}^{*}$ & $\begin{array}{c}x\left(0,0,0,0, t_{5}^{*}, s_{6}\right), \\
s_{6} \in \mathbb{F}_{q} \\
\end{array}$ & $q$ \\
\hline$x_{4}\left(t_{4}^{*}\right), t_{4}^{*} \in \mathbb{F}_{q^{3}}^{*}$ & $\begin{array}{c}x\left(0,0,0, t_{4}^{*}, s_{5}, s_{6}\right), \\
s_{5}, s_{6} \in \mathbb{F}_{q}\end{array}$ & $q^{2}$ \\
\hline $\begin{array}{l}x\left(0,0, t_{3}^{*}, 0, t_{5}, 0\right), \\
t_{3}^{*} \in \mathbb{F}_{q^{3}}^{*}, t_{5} \in \mathbb{F}_{q}\end{array}$ & $\begin{array}{c}x\left(0,0, t_{3}^{*}, s_{4}, \hat{s}_{5}, s_{6}\right), \\
s_{4} \in \mathbb{F}_{q^{3}}, s_{6} \in \mathbb{F}_{q}\end{array}$ & $q^{4}$ \\
\hline $\begin{array}{l}x\left(0, t_{2}^{*}, 0, t_{4}, t_{5}, 0\right), \\
t_{2}^{*} \in \mathbb{F}_{q}^{*}, t_{4}, t_{5} \in \mathbb{F}_{q}\end{array}$ & $\begin{array}{c}x\left(0, t_{2}^{*}, s_{3}, \hat{s}_{4}, \hat{s}_{5}, s_{6}\right), \\
s_{3} \in \mathbb{F}_{q^{3}}, s_{6} \in \mathbb{F}_{q}\end{array}$ & $q^{4}$ \\
\hline $\begin{array}{l}x\left(t_{1}^{*}, 0,0,0,0, t_{6}\right) \\
t_{1}^{*} \in \mathbb{F}_{q^{3}}^{*}, t_{6} \in \mathbb{F}_{q}\end{array}$ & $\begin{array}{c}x\left(t_{1}^{*}, 0, t_{1}^{*} s_{2}, s_{4}, s_{5}, \hat{s}_{6}\right), \\
s_{4} \in \mathbb{F}_{q^{3}}, s_{2}, s_{5} \in \mathbb{F}_{q}\end{array}$ & $q^{5}$ \\
\hline $\begin{array}{c}x\left(t_{1}^{*}, 0, \bar{t}_{3}^{*}, 0,0,0\right) \\
t_{1}^{*} \in \mathbb{F}_{q^{3}}^{*}, 0 \neq \bar{t}_{3}^{*} \in T^{t_{1}^{*}}\end{array}$ & $\begin{array}{c}x\left(t_{1}^{*}, 0, \bar{t}_{3}^{*}+t_{1}^{*} s_{2}, s_{4}, s_{5}, s_{6}\right) \\
s_{4} \in \mathbb{F}_{q^{3}}, s_{2}, s_{5}, s_{6} \in \mathbb{F}_{q}\end{array}$ & $q^{6}$ \\
\hline $\begin{array}{l}x\left(t_{1}^{*}, t_{2}^{*}, 0,0,0,0\right), \\
t_{1}^{*} \in \mathbb{F}_{q^{3}}^{*}, t_{2}^{*} \in \mathbb{F}_{q}^{*}\end{array}$ & $\begin{array}{c}x\left(t_{1}^{*}, t_{2}^{*}, s_{3}, s_{4}, s_{5}, s_{6}\right), \\
s_{3}, s_{4} \in \mathbb{F}_{q^{3}}, s_{5}, s_{6} \in \mathbb{F}_{q}\end{array}$ & $q^{8}$ \\
\hline
\end{tabular}

where $\hat{s}_{-}$is determined by some of $t_{-}^{*}, t_{-}$and $s_{-}$. The entries in the 1 st column are the representatives of the distinct conjugacy classes of $U$. For a fixed representative $x \in U,{ }^{U} x$ in the 2nd column is the conjugacy class of $x$, and $\left|{ }^{U} x\right|$ in the 3rd column is the number of the elements of ${ }^{U} x$.

(2) Let $\bar{t}_{3} \notin t_{1} \mathbb{F}_{q}^{+}$. By 3.8 ,

$$
\begin{aligned}
a_{1}= & t_{1}, \quad a_{2}=0, \quad a_{3}=\bar{t}_{3}+r_{2} t_{1}, \quad a_{4}=-r_{2} t_{1}^{q+1}-t_{1} r_{3}^{q}-t_{1}^{q} r_{3}+r_{1} \bar{t}_{3}^{q}+r_{1}^{q} \bar{t}_{3}, \\
a_{5}= & r_{2} t_{1}^{q^{2}+q+1}+\phi_{0}\left(r_{1}^{q^{2}}\left(-r_{3} t_{1}^{q}-r_{3}^{q} t_{1}\right)\right)+\phi_{0}\left(r_{3}^{q^{2}} t_{1}^{q+1}\right)+\phi_{0}\left(-t_{1} r_{4}^{q}\right)+\phi_{0}\left(r_{1}^{q^{2}+q} \bar{t}_{3}\right), \\
a_{6}= & t_{6}+r_{2}^{2} t_{1}^{q^{2}+q+1}+r_{2} a_{5}+\phi_{0}\left(-t_{1} r_{3}^{q^{2}+q}\right)+\phi_{0}\left(-r_{1} \bar{t}_{3}^{q^{2}+q}\right) \\
& +\phi_{0}\left(-\bar{t}_{3} r_{4}^{q}\right)+\phi_{0}\left(\bar{t}_{3}^{q^{2}}\left(r_{2} t_{1}^{q+1}+t_{1} r_{3}^{q}+t_{1}^{q} r_{3}\right)\right) .
\end{aligned}
$$

For the fixed $r_{1}, r_{2}, r_{3}$ and $a_{5}$, let

$$
T:=\left\{t_{1} r_{4}^{q} \mid r_{4} \in \mathbb{F}_{q^{3}} \text {, and } r_{1}, r_{2}, r_{3}, a_{5} \text { are fixed }\right\} .
$$

By 3.2, $|T|=q^{2}$. Let $x_{0} \in T$, then $T=x_{0}+\operatorname{ker} \phi_{0}$ and $\bar{t}_{3} r_{4}^{q} \in \frac{\bar{t}_{3} x_{0}}{t_{1}}+\frac{\bar{t}_{3}}{t_{1}} \operatorname{ker} \phi_{0}$. We know $\bar{t}_{3} \notin t_{1} \mathbb{F}_{q}^{+}$, so $\frac{\bar{t}_{3}}{t_{1}} \in \mathbb{F}_{q^{3}}^{+} \backslash \mathbb{F}_{q}^{+}$. Hence

$$
\left\{\phi_{0}\left(\bar{t}_{3} r_{4}^{q}\right) \mid t_{1} r_{4}^{q} \in T\right\}=\left\{\phi_{0}\left(\frac{\bar{t}_{3} x_{0}}{t_{1}}\right)+\phi_{0}(t) \mid t \in \frac{\bar{t}_{3}}{t_{1}} \operatorname{ker} \phi_{0}\right\} \stackrel{\underline{3.9}}{\underline{\underline{3}}} \mathbb{F}_{q}^{+} .
$$

Thus $a_{6}$ can be every element of $\mathbb{F}_{q}$ for the fixed $r_{1}, r_{2}, r_{3}$ and $a_{5}$. Therefore,

$$
U\left(x_{1}\left(t_{1}\right) x_{3}\left(\bar{t}_{3}\right)\right)=\left\{x\left(t_{1}, 0, \bar{t}_{3}+s_{2} t_{1}, s_{4}, s_{5}, s_{6}\right) \mid s_{4} \in \mathbb{F}_{q^{3}}, s_{2}, s_{5}, s_{6} \in \mathbb{F}_{q}\right\} .
$$

By 3.8, the other conjugacy classes are also determined. Then the proposition is obtained. 
3.12 Remark. If $p=2$, the map $\zeta_{u}$ in Lemma 3.6 is not surjective. Similarly, Corollary 3.7 does not hold either. Thus there are some differences between the classification of conjugacy classes of $U$ for $p=2$ and for $p>2$. Some results still hold when their proofs does not use the surjective property. Let $x:=x\left(t_{1}, t_{2}, t_{3}, t_{4}, t_{5}, t_{6}\right) \in U$. If $t_{1}=t_{3}=0$, the classifications of conjugacy classes of $x$ for $p=2$ and these for $p>2$ are the same. Otherwise, the analysis of conjugacy classes of $x$ for $p=2$ and this for $p>2$ are different. For example, let $t_{3}^{*} \in \mathbb{F}_{q^{3}}^{*}$, then $\left\{t_{3}^{*} r^{q}+t_{3}^{* q} r \mid r \in \mathbb{F}_{q^{3}}\right\}=$ $\left\{\zeta_{t_{3}^{*}}\left(r^{q^{2}}\right) \mid r \in \mathbb{F}_{q^{3}}\right\}=\operatorname{im} \zeta_{t_{3}^{*}}$ and $\left|\operatorname{im} \zeta_{t_{3}^{*}}\right|=q^{2}$. Similar to the proof of Proposition 3.11 we obtain the conjugacy class of $x_{3}\left(t_{3}^{*}\right)$ for $p=2$ as follows:

$$
{ }^{U} x_{3}\left(t_{3}^{*}\right)=\left\{\begin{array}{ll}
\left\{x\left(0,0, t_{3}^{*}, \tilde{s}_{4}, s_{5}, s_{6}\right) \in U \mid \tilde{s}_{4} \in \operatorname{im} \zeta_{t_{3}^{*}}, s_{5}, s_{6} \in \mathbb{F}_{q}\right\} & \text { if } p=2 \\
\left\{x\left(0,0, t_{3}^{*}, s_{4}, \hat{s}_{5}, s_{6}\right) \in U \mid s_{4} \in \mathbb{F}_{q^{3}}, s_{6} \in \mathbb{F}_{q}\right\} & \text { if } p>2
\end{array},\right.
$$

where $\hat{s}_{5} \in \mathbb{F}_{q}$ is determined by $t_{3}^{*}$ and $s_{4}$ (see Proposition 3.11).

\section{Irreducible characters}

In [Le16], Tung Le constructed and counted all ordinary irreducible characters of $U$, mainly using Clifford theory. In this section, we give the specific constructions of the irreducible characters of $U$ using Clifford's Theorem (see [CR81]) when $p>2$, so that we can determine the values of the irreducible characters in the next section.

Let $G$ be a finite group, $N$ a normal subgroup of $G$, and $K$ a field. Let $\operatorname{Irr}(G)$ be the set of all complex irreducible characters of $G$, and $\operatorname{triv}_{G}$ the trivial character of $G$. Let $H$ be a subgroup of $G, \chi \in \operatorname{Irr}(G)$ and $\lambda \in \operatorname{Irr}(H)$, then we denote by $\operatorname{Ind}_{H}^{G} \lambda$ the character induced from $\lambda$, and deonte by $\operatorname{Res}_{H}^{G} \chi$ the restriction of $\chi$ to $H$. The center of $G$ is denoted by $Z(G)$. The kernel of $\chi$ is ker $\chi:=\{g \in G \mid \chi(g)=\chi(1)\}$. The commutator subgroup of $G$ is $G^{\prime}=\langle[x, y] \mid x, y \in G\rangle$, where $[x, y]=x^{-1} y^{-1} x y$. Let $\lambda \in \operatorname{Irr}(N)$, then the inertia group in $G$ is $I_{G}(\lambda)=\left\{g \in G \mid \lambda^{g}=\lambda\right\}$ where $\lambda^{g}(n)=\lambda\left(g n g^{-1}\right)$ for all $n \in N$. In paricular, $N \unlhd I_{G}(\lambda) \leqslant G$.

4.1 Notation/Lemma. Let $\vartheta: \mathbb{F}_{q}^{+} \rightarrow \mathbb{C}^{*}$ denote a fixed nontrivial linear character of the additive group $\mathbb{F}_{q}^{+}$of $\mathbb{F}_{q}$ once and for all. In particular, $\sum_{x \in \mathbb{F}_{q}^{+}} \vartheta(x)=0$.

We determine the irreducible characters of $\mathbb{F}_{q^{3}}^{+}$and $\mathbb{F}_{q}^{+}$.

4.2 Lemma. Let $a \in \mathbb{F}_{q^{3}}, b \in \mathbb{F}_{q}$ and

$$
\begin{aligned}
& \vartheta_{a}: \mathbb{F}_{q^{3}}^{+} \rightarrow \mathbb{C}^{*}: x \mapsto \vartheta \pi_{q}(a x), \\
& \vartheta_{b}: \mathbb{F}_{q}^{+} \rightarrow \mathbb{C}^{*}: y \mapsto \vartheta \pi_{q}(b y)=\vartheta(b y),
\end{aligned}
$$

then $\operatorname{Irr}\left(\mathbb{F}_{q^{3}}^{+}\right)=\left\{\vartheta_{a} \mid a \in \mathbb{F}_{q^{3}}\right\}$ and $\operatorname{Irr}\left(\mathbb{F}_{q}^{+}\right)=\left\{\vartheta_{b} \mid b \in \mathbb{F}_{q}\right\}$.

Proof. Let $a \in \mathbb{F}_{q^{3}}$. By 3.5 and 4.1, we get $\vartheta_{a}(x+y)=\vartheta_{a}(x) \cdot \vartheta_{a}(y)$ for all $x, y \in \mathbb{F}_{q^{3}}$, so $\operatorname{Irr}\left(\mathbb{F}_{q^{3}}^{+}\right) \supseteq\left\{\vartheta_{a} \mid a \in \mathbb{F}_{q^{3}}\right\}$. Let $a, c \in \mathbb{F}_{q^{3}}$ and $\vartheta_{a}=\vartheta_{c}$, then $\vartheta_{a}(x)=\vartheta_{c}(x)$ for all $x \in \mathbb{F}_{q^{3}}$, i.e. $\vartheta \pi_{q}(a x)=\vartheta \pi_{q}(c x)$ for all $x \in \mathbb{F}_{q^{3}}$. Thus $a=c$ by 3.5. Otherwise, $\vartheta(y)=1$ for all $y \in \mathbb{F}_{q}$, this is a contradiction since $\vartheta$ is nontrivial by 4.1. Thus $\#\left\{\vartheta_{a} \mid a \in \mathbb{F}_{q^{3}}\right\}=q^{3}$. Therefore, $\operatorname{Irr}\left(\mathbb{F}_{q^{3}}^{+}\right)=\left\{\vartheta_{a} \mid a \in \mathbb{F}_{q^{3}}\right\}$. Similarly, we obtain the second formula.

4.3 Corollary. Let $u \in \mathbb{F}_{q^{3}}$, and $\tilde{\vartheta}_{u}: \mathbb{F}_{q^{3}}^{+} \rightarrow \mathbb{C}^{*}: t \mapsto \vartheta \pi_{q}\left(u^{q} t+u t^{q^{2}}\right)$, then $\tilde{\vartheta}_{u}=\vartheta_{\eta^{-1}\left(\eta+\eta^{q}\right) u^{q}}$ and $\operatorname{Irr}\left(\mathbb{F}_{q^{3}}^{+}\right)=\left\{\tilde{\vartheta}_{u} \mid u \in \mathbb{F}_{q^{3}}\right\}$.

Proof. For all $t \in \mathbb{F}_{q^{3}}^{+}, \tilde{\vartheta}_{u}(t)=\vartheta \pi_{q}\left(u^{q} t+u t^{q^{2}}\right) \stackrel{3.5}{=} \vartheta \phi_{0}\left(\eta u^{q} t+\eta u t^{q^{2}}\right) \stackrel{3.2}{=} \vartheta \phi_{0}\left(\left(\eta+\eta^{q}\right) u^{q} t\right)$ $=\vartheta \pi_{q}\left(\eta^{-1}\left(\eta+\eta^{q}\right) u^{q} t\right)=\vartheta_{\eta^{-1}\left(\eta+\eta^{q}\right) u^{q}}(t)$. By 3.4, $\eta+\eta^{q} \neq 0$. By 4.2, we obtain the second formula. 
4.4 Lemma. Let $G$ be a finite group, $Z(G) \subseteq N \unlhd G$, and $\chi \in \operatorname{Irr}(G)$. Let $\lambda \in \operatorname{Irr}(N)$ such that $\left\langle\operatorname{Res}_{N}^{G} \chi, \lambda\right\rangle_{N}=e>0$. Then for all $g \in Z(G)$,

$$
\left(\operatorname{Res}_{N}^{G} \chi\right)(g)=e \frac{|G|}{\left|I_{G}(\lambda)\right|} \lambda(g)
$$

and $g \notin \operatorname{ker} \chi \Longleftrightarrow g \notin \operatorname{ker} \lambda$. In particular, if $X \leqslant Z(G)$, then $X \nsubseteq \operatorname{ker} \chi$ if and only if $X \nsubseteq \operatorname{ker} \lambda$.

Proof. By Clifford's Theorem, we have for all $g \in Z(G)$

$$
\left(\operatorname{Res}_{N}^{G}\right) \chi(g)=e \sum_{h \in T_{I_{G}(\lambda) \backslash G}} \lambda^{h}(g) \stackrel{g \in Z(G)}{=} e \frac{|G|}{\left|I_{G}(\lambda)\right|} \lambda(g) .
$$

Let 1 be the identity element of $G$ and $g_{0} \in Z(G)$, then

$$
\chi\left(g_{0}\right) \neq \chi(1) \Longleftrightarrow\left(\operatorname{Res}_{N}^{G} \chi\right)\left(g_{0}\right) \neq\left(\operatorname{Res}_{N}^{G} \chi\right)(1) \Longleftrightarrow \lambda\left(g_{0}\right) \neq \lambda(1) .
$$

Thus $g \notin \operatorname{ker} \chi \Longleftrightarrow g \notin \operatorname{ker} \lambda$.

4.5 Definition (2.1, [Le16]). Let $\chi$ be an irreducible character of a group $G$. $\chi$ is said to be almost faithful if $Z(G) \nless \operatorname{ker} \chi$.

We determine some inertia groups.

4.6 Lemma. Let $T:=X_{2} X_{3} X_{4} X_{5} X_{6}, N:=X_{4} X_{5} X_{6}$ and $H:=X_{1} X_{4} X_{5} X_{6}$.

(1) The subgroup $N$ is abelian, $N \unlhd U, T \unlhd U$ and $H \leqslant U$ as follows:

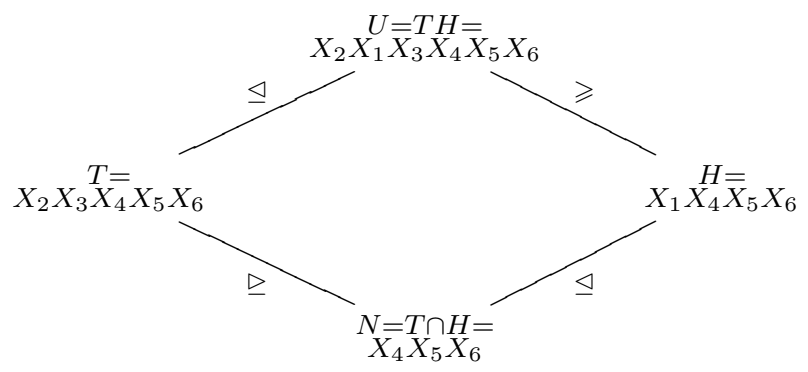

(2) Let $\lambda \in \operatorname{Irr}(N)$ and $\operatorname{Res}_{X_{6}}^{N} \lambda \neq \operatorname{triv}_{X_{6}}$. If $\lambda$ satisfies that $\operatorname{Res}_{X_{5}}^{N} \lambda=\operatorname{triv}_{X_{5}}$, we have

$$
I_{U}(\lambda)=\left\{u \in U \mid \lambda^{u}=\lambda\right\}=H .
$$

(3) Let $\lambda \in \operatorname{Irr}(N)$, the inertia group $I_{T}(\lambda)$ is

$$
I_{T}(\lambda)=\left\{\begin{array}{ll}
T & \text { if } \operatorname{Res}_{X_{6}}^{N} \lambda=\operatorname{triv}_{X_{6}} \\
N & \text { if } \operatorname{Res}_{X_{6}}^{N} \lambda \neq \operatorname{triv}_{X_{6}}
\end{array} .\right.
$$

(4) Let $\lambda \in \operatorname{Irr}(N)$, then the inertia group $I_{H}(\lambda)$ is

$$
I_{H}(\lambda)=\left\{\begin{array}{ll}
H & \text { if } \operatorname{Res}_{X_{5}}^{N} \lambda=\operatorname{triv}_{X_{5}} \\
N & \text { if } \operatorname{Res}_{X_{5}}^{N} \lambda \neq \operatorname{triv}_{X_{5}}
\end{array} .\right.
$$

(5) Let $\psi \in \operatorname{Irr}(T)$ and $X_{6}=Z(T) \nsubseteq \operatorname{ker} \psi$, then the inertia group $I_{U}(\psi)$ is

$$
I_{U}(\psi)=\left\{u \in U \mid \psi^{u}=\psi\right\}=U .
$$


Proof. (1) From the commutator relations of $U$ and by 3.8, we obtain that $N$ is abelian, $N$ and $T$ are normal subgroups of $U$, and $H$ is a subgroup of $U$. Then $N$ is a normal subgroup of $N$ and $H$, since $N$ is a normal subgroup of $U$.

(2) Let $u:=x\left(r_{1}, r_{2}, r_{3}, r_{4}, r_{5}, r_{6}\right) \in U, x:=x_{4}\left(t_{4}\right) x_{5}\left(t_{5}\right) x_{6}\left(t_{6}\right) \in N$, and $\lambda \in \operatorname{Irr}(N)$, then $\lambda$ is a linear character since $N$ is abelian. We have

$$
\begin{gathered}
\lambda^{u}(x)=\lambda\left(u \cdot x \cdot u^{-1}\right) \\
\stackrel{3.8}{=} \lambda\left(x_{4}\left(t_{4}\right) x_{5}\left(t_{5}+\phi_{0}\left(r_{1} t_{4}^{q}\right)\right) x_{6}\left(t_{6}+r_{2} t_{5}+\phi_{0}\left(r_{1} r_{2} t_{4}^{q}\right)+\phi_{0}\left(r_{3} t_{4}^{q}\right)\right)\right) \\
\stackrel{\operatorname{Res}_{X_{5}}^{N}{ }^{\lambda=} \operatorname{triv}_{X_{5}}}{=} \lambda\left(x_{4}\left(t_{4}\right)\right) \cdot \lambda\left(x_{6}\left(t_{6}+r_{2} t_{5}+\phi_{0}\left(r_{1} r_{2} t_{4}^{q}\right)+\phi_{0}\left(r_{3} t_{4}^{q}\right)\right)\right) .
\end{gathered}
$$

Since $X_{6} \nsubseteq$ ker $\lambda$ and $X_{5} \subseteq \operatorname{ker} \lambda$, we get

$$
\begin{gathered}
I_{U}(\lambda)=\left\{u \in U \mid \lambda^{u}=\lambda\right\}=\left\{u \in U \mid \lambda\left(u \cdot x \cdot u^{-1}\right)=\lambda(x) \text { for all } x \in N\right\} \\
\left.\quad 3 u:=x\left(r_{1}, r_{2}, r_{3}, r_{4}, r_{5}, r_{6}\right) \in U \mid r_{2}=r_{3}=0\right\}=X_{1} X_{4} X_{5} X_{6}=H .
\end{gathered}
$$

(3) c.f. the proof of (2).

(4) c.f. the proof of (2).

(5) Let $x_{6}\left(t_{6}\right) \in X_{6}$. Since $X_{6}=Z(T) \unlhd N \unlhd T$, there exists $\lambda \in \operatorname{Irr}(N)$ such that $0<e=$ $\left\langle\operatorname{Res}_{N}^{T} \psi, \lambda\right\rangle_{N}$, then $\left(\operatorname{Res}_{N}^{T} \psi\right)\left(x_{6}\left(t_{6}\right)\right)=e \frac{|T|}{\left|I_{T}(\lambda)\right|} \lambda\left(x_{6}\left(t_{6}\right)\right)$. By 4.4, $X_{6}=Z(T) \nsubseteq \operatorname{ker} \lambda$ since

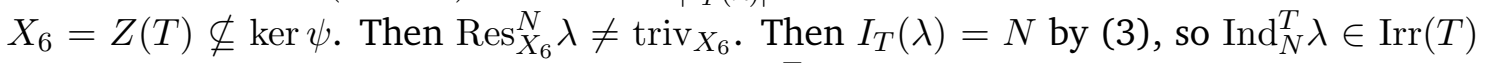
by Clifford's Theorem. Thus $e=1$ and $\psi=\operatorname{Ind}_{N}^{T} \lambda$.

Let $u:=x\left(s_{1}, s_{2}, s_{3}, s_{4}, s_{5}, s_{6}\right) \in U$ and $h:=x\left(0, t_{2}, t_{3}, t_{4}, t_{5}, t_{6}\right) \in T$, then

$$
\psi(h)=\left(\operatorname{Ind}_{N}^{T} \lambda\right)(h)=\frac{1}{|N|} \sum_{\substack{y \in T \\ y \cdot h \cdot y^{-1} \in N}} \lambda\left(y \cdot h \cdot y^{-1}\right) .
$$

- Let $t_{2} \neq 0$ or $t_{3} \neq 0$, then $\psi(h)=\left(\operatorname{Ind}_{N}^{T} \lambda\right)(h)=0$ and

$$
\left(\psi^{u}\right)(h)=\left(\operatorname{Ind}_{N}^{T} \lambda\right)^{u}(h)=\left(\operatorname{Ind}_{N}^{T} \lambda\right)\left(u \cdot h \cdot u^{-1}\right) \stackrel{3.8}{=} 0 .
$$

- Let $t_{2}=t_{3}=0$, then by 3.8

$$
\begin{gathered}
\psi(h)=\left(\operatorname{Ind}_{N}^{T} \lambda\right)(h)=\frac{1}{|N|} \sum_{\substack{y \in T \\
y \cdot h \cdot y^{-1} \in N}} \lambda\left(y \cdot h \cdot y^{-1}\right)=\frac{1}{|N|} \sum_{y \in T} \lambda\left(y \cdot h \cdot y^{-1}\right) \\
y:=x\left(0, r_{2}, r_{3}, r_{4}, r_{5}, r_{6}\right) \frac{1}{|N|} \sum_{\substack{r_{2}, r_{5}, r_{6} \in \mathbb{F}_{q} \\
r_{3}, r_{4} \in \mathbb{F}_{q^{3}}}} \lambda\left(x_{4}\left(t_{4}\right) x_{5}\left(t_{5}\right) x_{6}\left(t_{6}+r_{2} t_{5}+\phi_{0}\left(r_{3} t_{4}^{q}\right)\right)\right),
\end{gathered}
$$

and

$$
\begin{gathered}
\left(\psi^{u}\right)(h)=\left(\operatorname{Ind}_{N}^{T} \lambda\right)^{u}(h)=\left(\operatorname{Ind}_{N}^{T} \lambda\right)\left(u \cdot h \cdot u^{-1}\right) \\
=\left(\operatorname{Ind}_{N}^{T} \lambda\right)\left(x_{4}\left(t_{4}\right) x_{5}\left(t_{5}+\phi_{0}\left(s_{1} t_{4}^{q}\right)\right) x_{6}\left(t_{6}+s_{2} t_{5}+\phi_{0}\left(s_{1} s_{2} t_{4}^{q}\right)+\phi_{0}\left(s_{3} t_{4}^{q}\right)\right)\right) \\
=\frac{1}{|N|} \sum_{\substack{r_{2}, r_{5}, r_{6} \in \mathbb{F}_{q} \\
r_{3}, r_{4} \in \mathbb{F}_{q^{3}}}} \lambda\left(x_{4}\left(t_{4}\right) x_{5}\left(t_{5}+\phi_{0}\left(s_{1} t_{4}^{q}\right)\right) x_{6}\left(t_{6}+s_{2} t_{5}+\phi_{0}\left(s_{1} s_{2} t_{4}^{q}\right)+\phi_{0}\left(s_{3} t_{4}^{q}\right)\right)\right. \\
\left.\cdot x_{6}\left(r_{2} t_{5}+r_{2} \phi_{0}\left(s_{1} t_{4}^{q}\right)+\phi_{0}\left(r_{3} t_{4}^{q}\right)\right)\right) .
\end{gathered}
$$


Since $X_{6} \nsubseteq$ ker $\lambda$, we have for all $u \in U$

$$
\left(\psi^{u}\right)(h)=\psi(h)=\left\{\begin{array}{ll}
q^{4} \lambda\left(x_{6}\left(t_{6}\right)\right) & \text { if } t_{2}=t_{3}=t_{4}=t_{5}=0 \\
0 & \text { otherwise }
\end{array} .\right.
$$

Thus $I_{U}(\psi)=U$.

We determine the irreducible characters of the abelian group $X_{4} X_{5} X_{6}$. From now on, we set $N:=X_{4} X_{5} X_{6}$.

4.7 Lemma. Let $A_{17}, A_{16} \in \mathbb{F}_{q}, A_{15} \in \mathbb{F}_{q^{3}}$ and

$$
\lambda^{A_{17}, A_{16}, A_{15}}\left(x_{4}\left(t_{4}\right) x_{5}\left(t_{5}\right) x_{6}\left(t_{6}\right)\right):=\vartheta\left(A_{17} t_{6}\right) \cdot \vartheta\left(A_{16} t_{5}\right) \cdot \vartheta \pi_{q}\left(A_{15}^{q} t_{4}+A_{15} t_{4}^{q^{2}}\right),
$$

then $\operatorname{Irr}(N)=\left\{\lambda^{A_{17}, A_{16}, A_{15}} \mid A_{17}, A_{16} \in \mathbb{F}_{q}, A_{15} \in \mathbb{F}_{q^{3}}\right\}$.

Now we determine the irreducible characters of the subgroup $H=X_{1} X_{4} X_{5} X_{6}$ of $U$.

4.8 Lemma. Let $H:=X_{1} X_{4} X_{5} X_{6}$ and $\tilde{\chi} \in \operatorname{Irr}(H)$, then $H^{\prime}=X_{5}$.

(1) If $X_{5} \subseteq \operatorname{ker} \tilde{\chi}$, let $\bar{H}_{146}:=X_{5} \backslash H \cong \bar{X}_{1} \bar{X}_{4} \bar{X}_{6}, \bar{\chi}^{A_{17}, A_{15}, A_{12}} \in \operatorname{Irr}\left(\bar{H}_{146}\right)$,

$$
\bar{\chi}^{A_{17}, A_{15}, A_{12}}\left(\bar{x}_{1}\left(t_{1}\right) \bar{x}_{4}\left(t_{4}\right) \bar{x}_{6}\left(t_{6}\right)\right):=\vartheta\left(A_{17} t_{6}\right) \cdot \vartheta \pi_{q}\left(A_{15}^{q} t_{4}+A_{15} t_{4}^{q^{2}}\right) \cdot \vartheta \pi_{q}\left(A_{12} t_{1}\right) .
$$

Denote by $\tilde{\chi}^{A_{17}, A_{15}, A_{12}}$ the lift of $\bar{\chi}^{A_{17}, A_{15}, A_{12}}$ to $H$, then

$$
\operatorname{Irr}(H)_{1}:=\left\{\tilde{\chi} \in \operatorname{Irr}(H) \mid X_{5} \subseteq \operatorname{ker} \tilde{\chi}\right\}=\left\{\tilde{\chi}^{A_{17}, A_{15}, A_{12}} \in \operatorname{Irr}(H) \mid A_{17} \in \mathbb{F}_{q}, A_{15}, A_{12} \in \mathbb{F}_{q^{3}}\right\} .
$$

(2) If $X_{5} \nsubseteq$ ker $\tilde{\chi}$, we have

$$
\operatorname{Irr}(H)_{2}:=\left\{\tilde{\chi} \in \operatorname{Irr}(H) \mid X_{5} \nsubseteq \operatorname{ker} \tilde{\chi}\right\}=\left\{\operatorname{Ind}_{N}^{H} \lambda^{A_{17}, A_{16}^{*}, 0} \mid A_{17} \in \mathbb{F}_{q}, A_{16}^{*} \in \mathbb{F}_{q}^{*}\right\} .
$$

Thus, $\operatorname{Irr}(H)=\operatorname{Irr}(H)_{1} \cup \dot{\operatorname{Irr}}(H)_{2}$, i.e. $H$ has $q^{7}$ linear characters and $(q-1) q$ irreducible characters of degree $q^{3}$.

Proof. Let $\tilde{\chi} \in \operatorname{Irr}(H)$. From the commutator relations, $H^{\prime}=X_{5}$ and $Z(H)=X_{5} X_{6}$.

(1) If $X_{5} \subseteq \operatorname{ker} \tilde{\chi}, \tilde{\chi}$ is linear. Since $H^{\prime}=X_{5}$, all linear characters of $H$ are precisely the lifts to $H$ of the irreducible characters of the abelian quotient group $X_{5} \backslash H$.

(2) If $X_{5} \nsubseteq$ ker $\tilde{\chi}$, let $\lambda \in \operatorname{Irr}(N)$ and $\left\langle\operatorname{Res}_{N}^{H} \tilde{\chi}, \lambda\right\rangle_{N}>0$. Since $X_{5} \unlhd Z(H) \unlhd N \unlhd H$, by $4.4 X_{5} \nsubseteq \operatorname{ker} \lambda$ i.e. $\operatorname{Res}_{X_{5}}^{N} \lambda \neq \operatorname{triv}_{X_{5}}$, then $I_{H}(\lambda)=N$ by (4) of 4.6. By Clifford's Theorem, $\tilde{\chi}=\operatorname{Ind}_{N}^{H} \lambda$ and $\operatorname{deg}(\tilde{\chi})=q^{3}$. Then there exists $\lambda^{A_{17}, A_{16}^{*}, A_{15}} \in \operatorname{Irr}(N)$ such that $\lambda=\lambda^{A_{17}, A_{16}^{*}, A_{15}}$. We note that $X_{1}$ is a transversal of $N$ in $H$. Let $r_{1} \in \mathbb{F}_{q^{3}}$, for all $x_{4}\left(t_{4}\right) x_{5}\left(t_{5}\right) x_{6}\left(t_{6}\right) \in N$,

$$
\begin{aligned}
& \left(\lambda^{A_{17}, A_{16}^{*}, A_{15}}\right)^{x_{1}\left(r_{1}\right)}\left(x_{4}\left(t_{4}\right) x_{5}\left(t_{5}\right) x_{6}\left(t_{6}\right)\right) \\
= & \lambda^{A_{17}, A_{16}^{*}, A_{15}}\left(x_{1}\left(r_{1}\right) \cdot x_{4}\left(t_{4}\right) x_{5}\left(t_{5}\right) x_{6}\left(t_{6}\right) \cdot x_{1}\left(r_{1}\right)^{-1}\right) \\
= & \lambda^{A_{17}, A_{16}^{*}, A_{15}}\left(x_{4}\left(t_{4}\right) x_{5}\left(t_{5}+\phi_{0}\left(r_{1} t_{4}^{q}\right)\right) x_{6}\left(t_{6}\right)\right) \\
= & \vartheta\left(A_{17} t_{6}\right) \cdot \vartheta\left(A_{16}^{*}\left(t_{5}+\phi_{0}\left(r_{1} t_{4}^{q}\right)\right)\right) \cdot \vartheta \pi_{q}\left(A_{15}^{q} t_{4}+A_{15} t_{4}^{q^{2}}\right) \\
= & \vartheta\left(A_{17} t_{6}\right) \cdot \vartheta\left(A_{16}^{*} t_{5}\right) \cdot \vartheta \pi_{q}\left(\eta^{-1}\left(\left(\eta+\eta^{q}\right) A_{15}^{q}+A_{16}^{*} r_{1}^{q^{2}}\right) t_{4}\right) .
\end{aligned}
$$

By Clifford's Theorem, $\tilde{\chi}=\operatorname{Ind}_{N}^{H} \lambda^{A_{17}, A_{16}^{*}, 0}$ and

$$
\operatorname{Res}_{N}^{H} \operatorname{Ind}_{N}^{H} \lambda^{A_{17}, A_{16}^{*}, 0}=\sum_{r_{1} \in \mathbb{F}_{q^{3}}}\left(\lambda^{A_{17}, A_{16}^{*}, A_{15}}\right)^{x_{1}\left(r_{1}\right)}=\sum_{B_{15} \in \mathbb{F}_{q^{3}}} \lambda^{A_{17}, A_{16}^{*}, B_{15}} .
$$


Thus $H$ has $q^{7}$ linear characters and $(q-1) q$ irreducible characters of degree $q^{3}$.

By the commutator relations, we determine the conjugacy classes of $H$, and obtain the character table of $H$.

4.9 Corollary. Let $x:=x\left(t_{1}, 0,0, t_{4}, t_{5}, t_{6}\right) \in H$ be a representative of one conjugacy class of $H$, then the character table of $H$ is shown in Table 2

Table 2: Character table of $H$

\begin{tabular}{c|ccc}
$\left|{ }^{H} x\right|$ & 1 & \multicolumn{1}{c}{$q$} & $q$ \\
$x$ & $x_{5}\left(t_{5}\right) x_{6}\left(t_{6}\right)$ & $x_{4}\left(t_{4}^{*}\right) x_{6}\left(t_{6}\right)$ & $x_{1}\left(t_{1}^{*}\right) x_{4}\left(t_{4}\right) x_{6}\left(t_{6}\right)$ \\
\hline \multirow{2}{*}{$\tilde{\chi}^{A_{17}, A_{15}, A_{12}}$} & $\vartheta\left(A_{17} t_{6}\right)$ & $\vartheta\left(A_{17} t_{6}\right)$ & $\vartheta\left(A_{17} t_{6}\right)$ \\
& & $\cdot \vartheta \pi_{q}\left(A_{15}^{q} t_{4}^{*}+A_{15} t_{4}^{* q^{2}}\right)$ & $\cdot \vartheta \pi_{q}\left(A_{15}^{q} t_{4}+A_{15} t_{4}^{q^{2}}\right)$ \\
& & & $\cdot \vartheta \pi_{q}\left(A_{12} t_{1}^{*}\right)$ \\
$\operatorname{Ind}_{N}^{H} \lambda^{A_{17}, A_{16}^{*}, 0}$ & $q^{3} \vartheta\left(A_{17} t_{6}\right)$ & 0 & 0 \\
& $\cdot \vartheta\left(A_{16}^{*} t_{5}\right)$ & &
\end{tabular}

Similar to $H$, we construct the irreducible character of the normal subgroup $X_{2} X_{3} X_{4} X_{5} X_{6}$ of $U$.

4.10 Lemma. Let $T:=X_{2} X_{3} X_{4} X_{5} X_{6}$ and $\psi \in \operatorname{Irr}(T)$, then $T^{\prime}=X_{6}$.

(1) If $X_{6} \subseteq \operatorname{ker} \psi$, let $\bar{H}_{2345}:=X_{6} \backslash T \cong \bar{X}_{2} \bar{X}_{3} \bar{X}_{4} \bar{X}_{5}, \bar{\chi}^{A_{16}, A_{15}, A_{13}, A_{23}} \in \operatorname{Irr}\left(\bar{H}_{2345}\right)$,

$$
\begin{aligned}
& \bar{\chi}^{A_{16}, A_{15}, A_{13}, A_{23}}\left(\bar{x}_{2}\left(t_{2}\right) \bar{x}_{3}\left(t_{3}\right) \bar{x}_{4}\left(t_{4}\right) \bar{x}_{5}\left(t_{5}\right)\right) \\
& :=\vartheta\left(A_{16} t_{5}\right) \cdot \vartheta \pi_{q}\left(A_{15}^{q} t_{4}+A_{15} t_{4}^{q^{2}}\right) \cdot \vartheta \pi_{q}\left(-A_{13} t_{3}\right) \cdot \vartheta\left(A_{23} t_{2}\right) .
\end{aligned}
$$

Denote by $\psi^{A_{16}, A_{15}, A_{13}, A_{23}}$ the lift of $\bar{\chi}^{A_{16}, A_{15}, A_{13}, A_{23}}$ to $T$, then

$$
\operatorname{Irr}(T)_{1}:=\left\{\psi \in \operatorname{Irr}(T) \mid X_{6} \subseteq \operatorname{ker} \psi\right\}=\left\{\psi^{A_{16}, A_{15}, A_{13}, A_{23}} \mid A_{16}, A_{23} \in \mathbb{F}_{q}, A_{13}, A_{15} \in \mathbb{F}_{q^{3}}\right\}
$$

(2) If $X_{6} \nsubseteq \operatorname{ker} \psi$, let $\psi^{A_{17}^{*}}:=\operatorname{Ind}_{N}^{T} \lambda^{A_{17}^{*}, 0,0}$. Then

$$
\operatorname{Irr}(T)_{2}:=\left\{\psi \in \operatorname{Irr}(T) \mid X_{6} \not \subseteq \operatorname{ker} \psi\right\}=\left\{\psi^{A_{17}^{*}} \mid A_{17} \in \mathbb{F}_{q}^{*}\right\}
$$

Thus, $\operatorname{Irr}(T)=\operatorname{Irr}(T)_{1} \cup \dot{U} \operatorname{Irr}(T)_{2}$, i.e. $T$ has $q^{8}$ linear characters and $(q-1)$ irreducible characters of degree $q^{4}$.

By the commutator relations, we also determine the conjugacy classes of $T$ and the character table of $T$.

4.11 Corollary. Let $x:=x\left(0, t_{2}, t_{3}, t_{4}, t_{5}, t_{6}\right) \in T$ be a representative of one conjugacy class of $T$, then the character table of $T$ is the one in Table 3.

Now we give the explicit constructions of the irreducible characters of $U$ when $p>2$.

4.12 Proposition. Let $p>2, A_{12}, A_{13}, A_{15} \in \mathbb{F}_{q^{3}}$, and $A_{23}, A_{16}, A_{17} \in \mathbb{F}_{q}$.

Let $A_{12}^{*}, A_{13}^{*}, A_{15}^{*} \in \mathbb{F}_{q^{3}}^{*}, A_{23}^{*}, A_{16}^{*}, A_{17}^{*} \in \mathbb{F}_{q}^{*}$ and $T^{A_{13}^{*}}$ be a transversal of $A_{13}^{*} \mathbb{F}_{q}^{+}$in $\mathbb{F}_{q^{3}}^{+}$. Then all of the pairwise orthogonal irreducible characters of $U$ can be constructed as follows: 
Table 3: Character table of $T$

\begin{tabular}{c|ccccc}
$\left|{ }^{T} x\right|$ & 1 & $q$ & $q$ & $q$ & $q$ \\
$x$ & $x_{6}\left(t_{6}\right)$ & $x_{5}\left(t_{5}^{*}\right)$ & $x_{4}\left(t_{4}^{*}\right) x_{5}\left(t_{5}\right)$ & $x_{3}\left(t_{3}^{*}\right) x_{4}\left(t_{4}\right) x_{5}\left(t_{5}\right)$ & $x_{2}\left(t_{2}^{*}\right) x_{3}\left(t_{3}\right)$ \\
$\cdot x_{4}\left(t_{4}\right) x_{5}\left(t_{5}\right)$
\end{tabular}

(1) Let $\bar{U}:=X_{3} X_{4} X_{5} X_{6} \backslash U=\bar{X}_{2} \bar{X}_{1}, \bar{\chi}_{\text {lin }}^{A_{12}, A_{23}} \in \operatorname{Irr}(\bar{U}), \bar{\chi}_{\text {lin }}^{A_{12}, A_{23}}\left(\bar{x}_{2}\left(t_{2}\right) \bar{x}_{1}\left(t_{1}\right)\right):=\vartheta \pi_{q}\left(A_{12} t_{1}\right)$. $\vartheta\left(A_{23} t_{2}\right)$, and denote by $\chi_{\text {lin }}^{A_{12}, A_{23}}$ the lift of $\bar{\chi}_{\text {lin }}^{A_{12}, A_{23}}$ to $U$, then

$$
\mathfrak{F}_{l i n}:=\left\{\chi \in \operatorname{Irr}(U) \mid X_{3} X_{4} X_{5} X_{6} \subseteq \operatorname{ker} \chi\right\}=\left\{\chi_{\text {lin }}^{A_{12}, A_{23}} \mid A_{12} \in \mathbb{F}_{q^{3}}, A_{23} \in \mathbb{F}_{q}\right\} .
$$

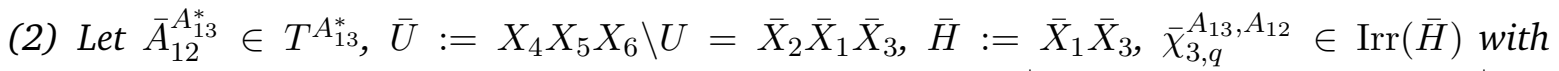
$\bar{\chi}_{3, q}^{A_{13}, A_{12}}\left(\bar{x}_{1}\left(t_{1}\right) \bar{x}_{3}\left(t_{3}\right)\right):=\vartheta \pi_{q}\left(A_{12} t_{1}-A_{13} t_{3}\right)$. Let $\chi_{3, q}^{A_{13}^{*}, A_{12}^{A_{13}^{*}}}$ be the lift of $\operatorname{Ind}_{\bar{H}}^{\bar{U}} \bar{\chi}_{3, q}^{A_{13}^{*}, \bar{A}_{12}^{A_{13}^{*}}}$ to $U$, then

$\mathfrak{F}_{3}:=\left\{\chi \in \operatorname{Irr}(U) \mid X_{4} X_{5} X_{6} \subseteq \operatorname{ker} \chi, X_{3} \nsubseteq \mathbb{k e r} \chi\right\}=\left\{\chi_{3, q}^{A_{13}^{*}, \bar{A}_{12}^{A_{13}^{*}}} \mid A_{13}^{*} \in \mathbb{F}_{q^{3}}^{*}, \bar{A}_{12}^{A_{13}^{*}} \in T^{A_{13}^{*}}\right\}$.

(3) Let $\bar{U}:=X_{5} X_{6} \backslash U=\bar{X}_{2} \bar{X}_{1} \bar{X}_{3} \bar{X}_{4}, \bar{H}:=\bar{X}_{2} \bar{X}_{3} \bar{X}_{4}, \bar{\chi}_{4, q^{3}}^{A_{15}, A_{23}, A_{13}} \in \operatorname{Irr}(\bar{H})$, and

$$
\bar{\chi}_{4, q^{3}}^{A_{15}, A_{23}, A_{13}}\left(\bar{x}_{2}\left(t_{2}\right) \bar{x}_{3}\left(t_{3}\right) \bar{x}_{4}\left(t_{4}\right)\right):=\vartheta\left(A_{23} t_{2}\right) \cdot \vartheta \pi_{q}\left(-A_{13} t_{3}\right) \cdot \vartheta \pi_{q}\left(A_{15}^{q} t_{4}+A_{15} t_{4}^{q^{2}}\right) .
$$

Denote by $\chi_{4, q^{3}}^{A_{15}^{*}, A_{23}}$ the lift of $\operatorname{Ind}_{\bar{H}} \bar{U} \bar{\chi}_{4, q^{3}}^{A_{15}^{*}, A_{23}, 0}$ to $U$, then

$$
\mathfrak{F}_{4}:=\left\{\chi \in \operatorname{Irr}(U) \mid X_{5} X_{6} \subseteq \operatorname{ker} \chi, X_{4} \nsubseteq \subseteq \operatorname{ker} \chi\right\}=\left\{\chi_{4, q^{3}}^{A_{15}^{*}, A_{23}} \mid A_{15}^{*} \in \mathbb{F}_{q^{3}}^{*}, A_{23} \in \mathbb{F}_{q}\right\} .
$$

(4) Let $\bar{U}:=X_{6} \backslash U=\bar{X}_{2} \bar{X}_{1} \bar{X}_{3} \bar{X}_{4} \bar{X}_{5}, \bar{H}:=\bar{X}_{2} \bar{X}_{3} \bar{X}_{4} \bar{X}_{5}, \bar{\chi}_{5, q^{3}}^{A_{16}, A_{23}, A_{13}, A_{15}} \in \operatorname{Irr}(\bar{H})$, and

$$
\begin{aligned}
& \bar{\chi}_{5, q^{3}}^{A_{16}, A_{23}, A_{13}, A_{15}}\left(\bar{x}_{2}\left(t_{2}\right) \bar{x}_{3}\left(t_{3}\right) \bar{x}_{4}\left(t_{4}\right) \bar{x}_{5}\left(t_{5}\right)\right) \\
:= & \vartheta\left(A_{23} t_{2}\right) \cdot \vartheta \pi_{q}\left(-A_{13} t_{3}\right) \cdot \vartheta \pi_{q}\left(A_{15}^{q} t_{4}+A_{15} t_{4}^{q^{2}}\right) \cdot \vartheta\left(A_{16} t_{5}\right) .
\end{aligned}
$$

Denote by $\chi_{5, q^{3}}^{A_{16}^{*}, A_{23}, A_{13}}$ the lift of $\operatorname{Ind}_{\bar{H}}^{\bar{U}} \bar{\chi}_{5, q^{3}}^{A_{16}^{*}, A_{23}, A_{13}, 0}$ to $U$, then $\mathfrak{F}_{5}:=\left\{\chi \in \operatorname{Irr}(U) \mid X_{6} \subseteq \operatorname{ker} \chi, X_{5} \nsubseteq \subseteq \operatorname{ker} \chi\right\}=\left\{\chi_{5, q^{3}}^{A_{16}^{*}, A_{23}, A_{13}} \mid A_{16}^{*} \in \mathbb{F}_{q}^{*}, A_{23} \in \mathbb{F}_{q}, A_{13} \in \mathbb{F}_{q^{3}}\right\}$

(5) Let $H:=X_{1} X_{4} X_{5} X_{6}, \bar{H}:=X_{4} X_{5} \backslash H \cong \bar{X}_{1} \bar{X}_{6}, \bar{\chi}_{6, q^{4}}^{A_{17}, A_{12}} \in \operatorname{Irr}(\bar{H})$, and

$$
\bar{\chi}_{6, q^{4}}^{A_{17}, A_{12}}\left(\bar{x}_{1}\left(t_{1}\right) \bar{x}_{6}\left(t_{6}\right)\right):=\vartheta \pi_{q}\left(A_{12} t_{1}\right) \cdot \vartheta\left(A_{17} t_{6}\right) .
$$

Let $\tilde{\chi}_{6, q^{4}}^{A_{17}, A_{12}}$ denote the lift of $\bar{\chi}_{6, q^{4}}^{A_{17}, A_{12}}$ from $\bar{H}$ to $H$, and $\chi_{6, q^{4}}^{A_{17}^{*}, A_{12}}:=\operatorname{Ind}_{H}^{U} \tilde{\chi}_{6, q^{4}}^{A_{17}^{*}, A_{12}}$, then $\mathfrak{F}_{6}:=\left\{\chi \in \operatorname{Irr}(U) \mid X_{6} \nsubseteq \mathbb{k e r} \chi\right\}=\left\{\chi_{6, q^{4}}^{A_{17}^{*}, A_{12}} \mid A_{17}^{*} \in \mathbb{F}_{q}^{*}, A_{12} \in \mathbb{F}_{q^{3}}\right\}$. 
Hence $\operatorname{Irr}(U)=\mathfrak{F}_{\text {lin }} \cup \dot{\cup} \mathfrak{F}_{3} \dot{\cup} \mathfrak{F}_{4} \dot{\cup} \mathfrak{F}_{5} \dot{\cup} \mathfrak{F}_{6}$.

Proof. Let $\chi \in \operatorname{Irr}(U)$.

(1) Family $\mathfrak{F}_{\text {lin }}$, where $X_{3} X_{4} X_{5} X_{6} \subseteq \operatorname{ker} \chi$.

Since $U^{\prime}=X_{3} X_{4} X_{5} X_{6}$, all linear characters of $U$ are precisely the lifts of the linear characters of the quotient group $X_{3} X_{4} X_{5} X_{6} \backslash U$.

(2) Family $\mathfrak{F}_{3}$, where $X_{4} X_{5} X_{6} \subseteq \operatorname{ker} \chi$ and $X_{3} \nsubseteq \subseteq$ ker $\chi$.

Let $\bar{U}:=X_{4} X_{5} X_{6} \backslash U=\bar{X}_{2} \bar{X}_{1} \bar{X}_{3}$, then the commutator relation in $\bar{U}$ is $\left[\bar{x}_{2}\left(t_{2}\right), \bar{x}_{1}\left(t_{1}\right)\right]=$ $\bar{x}_{3}\left(t_{2} t_{1}\right)$. Let $\bar{H}:=\bar{X}_{1} \bar{X}_{3}, \bar{\chi}_{3, q}^{A_{13}, A_{12}} \in \operatorname{Irr}(\bar{H})$ and

$$
\bar{\chi}_{3, q}^{A_{13}, A_{12}}\left(\bar{x}_{1}\left(t_{1}\right) \bar{x}_{3}\left(t_{3}\right)\right):=\vartheta \pi_{q}\left(A_{12} t_{1}\right) \cdot \vartheta \pi_{q}\left(-A_{13} t_{3}\right) .
$$

We note that $\bar{X}_{2}$ is a transversal of $\bar{H}$ in $\bar{U}$, and $Z(\bar{U})=\bar{X}_{3}$. For all $s_{2} \in \mathbb{F}_{q}$,

$$
\begin{aligned}
& \left(\bar{\chi}_{3, q}^{A_{13}^{*}, A_{12}}\right)^{\bar{x}_{2}\left(s_{2}\right)}\left(\bar{x}_{1}\left(t_{1}\right) \bar{x}_{3}\left(t_{3}\right)\right)=\bar{\chi}_{3, q}^{A_{13}^{*}, A_{12}}\left(\bar{x}_{2}\left(s_{2}\right) \cdot \bar{x}_{1}\left(t_{1}\right) \bar{x}_{3}\left(t_{3}\right) \cdot \bar{x}_{2}\left(s_{2}\right)^{-1}\right) \\
= & \bar{\chi}_{3, q}^{A_{13}^{*}, A_{12}}\left(\bar{x}_{1}\left(t_{1}\right) \bar{x}_{3}\left(t_{3}+s_{2} t_{1}\right)\right)=\vartheta \pi_{q}\left(A_{12} t_{1}-A_{13}^{*}\left(t_{3}+s_{2} t_{1}\right)\right) \\
= & \vartheta \pi_{q}\left(\left(A_{12}-s_{2} A_{13}^{*}\right) t_{1}-A_{13}^{*} t_{3}\right)=\vartheta \pi_{q}\left(\left(A_{12}-s_{2} A_{13}^{*}\right) t_{1}\right) \cdot \vartheta \pi_{q}\left(-A_{13}^{*} t_{3}\right),
\end{aligned}
$$

so $I_{\bar{U}}\left(\bar{\chi}_{3, q}^{A_{13}^{*}, A_{12}}\right)=\bar{H}$. By Clifford theory, $\tilde{\chi}_{3, q}^{A_{13}^{*}, A_{12}}:=\operatorname{Ind}_{\bar{H}} \overline{\chi_{3, q}} \bar{\chi}_{33}^{*}, A_{12} \in \operatorname{Irr}(\bar{U})$ and

$$
\operatorname{Res}_{\bar{H}}^{\bar{U}} \tilde{\chi}_{3, q}^{A_{13}^{*}, A_{12}}=\sum_{s_{2} \in \mathbb{F}_{q}}\left(\bar{\chi}_{3, q}^{A_{13}^{*}, A_{12}}\right)^{\bar{x}_{2}\left(s_{2}\right)}=\sum_{s_{2} \in \mathbb{F}_{q}} \bar{\chi}_{3, q}^{A_{13}^{*}, A_{12}-s_{2} A_{13}^{*}} .
$$

By Clifford theory, there are $q^{2}\left(q^{3}-1\right)$ almost faithful irreducible characters of $\bar{U}$, i.e.

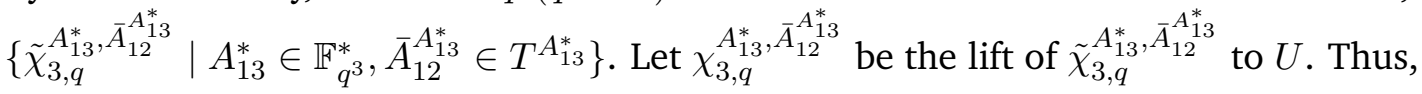
$\mathfrak{F}_{3}=\left\{\chi \in \operatorname{Irr}(U) \mid X_{4} X_{5} X_{6} \subseteq \operatorname{ker} \chi, X_{3} \nsubseteq \operatorname{ker} \chi\right\}=\left\{\chi_{3, q}^{A_{13}^{*}, \bar{A}_{12}^{A_{13}^{*}}} \mid A_{13}^{*} \in \mathbb{F}_{q^{3}}^{*}, \bar{A}_{12}^{A_{13}^{*}} \in T^{A_{13}^{*}}\right\}$.

(3) The proof of the Family $\mathfrak{F}_{4}$ is an adaptation of the proof of the Family $\mathfrak{F}_{3}$.

(4) The proof of the Family $\mathfrak{F}_{5}$ is also an adaptation of the proof of the Family $\mathfrak{F}_{3}$.

(5) Family $\mathfrak{F}_{6}$, where $X_{6} \nsubseteq$ ker $\chi$.

Let $T:=X_{2} X_{3} X_{4} X_{5} X_{6}, N:=X_{4} X_{5} X_{6}$, and $\chi \in \operatorname{Irr}(U)$ such that $X_{6} \nsubseteq \operatorname{ker}(\chi)$, then $Z(T)=Z(U)=X_{6}$. Let $\psi \in \operatorname{Irr}(T)$ and $\left\langle\psi, \operatorname{Res}_{T}^{U} \chi\right\rangle_{T}>0$, then $X_{6} \nsubseteq \operatorname{ker} \psi$ by 4.4. Let $\lambda^{A_{17}, A_{16}, A_{15}} \in \operatorname{Irr}(N)$ and

$$
\lambda^{A_{17}, A_{16}, A_{15}}\left(x_{4}\left(t_{4}\right) x_{5}\left(t_{5}\right) x_{6}\left(t_{6}\right)\right):=\vartheta\left(A_{17} t_{6}\right) \cdot \vartheta\left(A_{16} t_{5}\right) \cdot \vartheta \pi_{q}\left(A_{15}^{q} t_{4}+A_{15} t_{4}^{q^{2}}\right),
$$

then by 4.10, $\left\{\psi \in \operatorname{Irr}(T) \mid X_{6} \nsubseteq \mathbb{k e r} \psi\right\}=\left\{\operatorname{Ind}_{N}^{T} \lambda^{A_{17}^{*}, 0,0} \mid A_{17}^{*} \in \mathbb{F}_{q}^{*}\right\}=\left\{\psi^{A_{17}^{*}} \mid A_{17}^{*} \in \mathbb{F}_{q}^{*}\right\}$. By (5) of 4.6, $I_{U}\left(\psi^{A_{17}^{*}}\right)=U$, so $\operatorname{Res}_{T}^{U} \chi=z^{*} \psi^{A_{17}^{*}}$ for some $z^{*} \in \mathbb{N}^{*}$. Thus

$$
\begin{aligned}
& \mathfrak{F}_{6}=\left\{\chi \in \operatorname{Irr}(U) \mid X_{6} \nsubseteq \subseteq \operatorname{ker} \chi\right\}=\bigcup_{\substack{\psi \in \operatorname{Irr}(T) \\
X_{6} \nsubseteq \operatorname{ker} \psi}}\left\{\chi \in \operatorname{Irr}(U) \mid\left\langle\chi, \operatorname{Ind}_{T}^{U} \psi\right\rangle_{U}>0\right\} \\
& =\bigcup_{A_{17}^{*} \in \mathbb{F}_{q}^{*}}\left\{\chi \in \operatorname{Irr}(U) \mid\left\langle\chi, \operatorname{Ind}_{T}^{U} \psi^{\left.\left.A_{17}^{*}\right\rangle_{U}>0\right\}}\right.\right. \\
& =\bigcup_{A_{17}^{*} \in \mathbb{F}_{q}^{*}}\left\{\chi \in \operatorname{Irr}(U) \mid\left\langle\chi, \operatorname{Ind}_{N}^{U} \lambda^{A_{17}^{*}, 0,0}\right\rangle_{U}>0\right\} .
\end{aligned}
$$


Let $H:=X_{1} X_{4} X_{5} X_{6}$, then $H^{\prime}=X_{5}$ and $Z(H)=X_{4} X_{5} \unlhd H$. Let $\tilde{\chi}^{A_{17}, A_{15}, A_{12}} \in \operatorname{Irr}(H)$ as in (1) of 4.8. For all $x_{4}\left(t_{4}\right) x_{5}\left(t_{5}\right) x_{6}\left(t_{6}\right) \in N$,

$$
\begin{aligned}
& \left(\operatorname{Res}_{N}^{H} \tilde{\chi}^{A_{17}^{*}, 0, A_{12}}\right)\left(x_{4}\left(t_{4}\right) x_{5}\left(t_{5}\right) x_{6}\left(t_{6}\right)\right)=\tilde{\chi}^{A_{17}^{*}, 0, A_{12}}\left(x_{4}\left(t_{4}\right) x_{5}\left(t_{5}\right) x_{6}\left(t_{6}\right)\right) \\
= & \bar{\chi}\left(\bar{x}_{4}\left(t_{4}\right) \bar{x}_{6}\left(t_{6}\right)\right)=\vartheta\left(A_{17}^{*} t_{6}\right)=\lambda^{A_{17}^{*}, 0,0}\left(x_{4}\left(t_{4}\right) x_{5}\left(t_{5}\right) x_{6}\left(t_{6}\right)\right) .
\end{aligned}
$$

Thus $\operatorname{Res}_{N}^{H} \tilde{\chi}^{A_{17}^{*}, 0, A_{12}}=\lambda^{A_{17}^{*}, 0,0}$ for all $A_{12} \in \mathbb{F}_{q^{3}}$. By (4) of 4.6 we get $I_{H}\left(\lambda^{A_{17}^{*}, 0,0}\right)=H$, thus $\operatorname{Ind}_{N}^{H} \lambda^{A_{17}^{*}, 0,0}=\sum_{A_{12} \in \mathbb{F}_{q^{3}}} \tilde{\chi}^{A_{17}^{*}, 0, A_{12}}$. By (2) of $4.6 I_{U}\left(\lambda^{A_{17}^{*}, 0,0}\right)=H$. Then by Clifford's Theorem, $\operatorname{Ind}_{H}^{U} \tilde{\chi}^{A_{17}^{*}, 0, A_{12}} \in \operatorname{Irr}(U)$ for all $A_{17}^{*} \in \mathbb{F}_{q}^{*}$. Thus

$$
\begin{aligned}
\mathfrak{F}_{6} & =\bigcup_{A_{17}^{*} \in \mathbb{F}_{q}^{*}}\left\{\chi \in \operatorname{Irr}(U) \mid\left\langle\chi, \operatorname{Ind}_{H}^{U} \operatorname{Ind}_{N}^{H} \lambda^{A_{17}^{*}, 0,0}\right\rangle_{U}>0\right\} \\
= & \bigcup_{A_{17}^{*} \in \mathbb{F}_{q}^{*}}\left\{\chi \in \operatorname{Irr}(U) \mid\left\langle\chi, \operatorname{Ind}_{H}^{U} \tilde{\chi}^{A_{17}^{*}, 0, A_{12}}\right\rangle_{U}>0\right\} \\
& =\left\{\operatorname{Ind}_{H}^{U} \tilde{\chi}^{A_{17}^{*}, 0, A_{12}} \mid A_{17}^{*} \in \mathbb{F}_{q}^{*}, A_{12} \in \mathbb{F}_{q^{3}}\right\} .
\end{aligned}
$$

For $A_{17}^{*} \in \mathbb{F}_{q}^{*}$ and $A_{12} \in \mathbb{F}_{q^{3}}, X_{4} X_{5} \subseteq \operatorname{ker}\left(\tilde{\chi}^{A_{17}^{*}, 0, A_{12}}\right)$ and $X_{4} X_{5} \unlhd H$, so $\tilde{\chi}^{A_{17}^{*}, 0, A_{12}}$ is the lift to $H$ of some irreducible character of $\bar{H}:=X_{4} X_{5} \backslash H \cong \bar{X}_{1} \bar{X}_{6}$. Let $\bar{\chi}_{6, q^{4}}^{A_{11}^{*}, A_{12}} \in \operatorname{Irr}(\bar{H})$, and $\bar{\chi}_{6, q^{4}}^{A_{17}^{*}, A_{12}}\left(\bar{x}_{1}\left(t_{1}\right) \bar{x}_{6}\left(t_{6}\right)\right):=\vartheta \pi_{q}\left(A_{12} t_{1}\right) \cdot \vartheta\left(A_{17}^{*} t_{6}\right)$. Let $\tilde{\chi}_{6, q^{4}}^{A_{17}^{*}, A_{12}}$ denote the lift of $\bar{\chi}_{6, q^{4}}^{A_{17}^{*}, A_{12}}$ from $\bar{H}$ to $H$, then $\tilde{\chi}_{6, q^{4}}^{A_{17}^{*}, A_{12}}=\tilde{\chi}^{A_{17}^{*}, 0, A_{12}}$. Let $\chi_{6, q^{4}}^{A_{17}^{*}, A_{12}}:=\operatorname{Ind}_{H}^{U} \tilde{\chi}_{6, q^{4}}^{A_{17}^{*}, A_{12}}$, then

$$
\mathfrak{F}_{6}=\left\{\operatorname{Ind}_{H}^{U} \tilde{\chi}^{A_{17}^{*}, 0, A_{12}} \mid A_{17}^{*} \in \mathbb{F}_{q}^{*}, A_{12} \in \mathbb{F}_{q^{3}}\right\}=\left\{\chi_{6, q^{4}}^{A_{17}^{*}, A_{12}} \mid A_{17}^{*} \in \mathbb{F}_{q}^{*}, A_{12} \in \mathbb{F}_{q^{3}}\right\} .
$$

4.13 Remark. From the proof (5) of Propositon 4.12 we obtain that

$$
\begin{aligned}
& \operatorname{Ind}_{T}^{U} \psi^{A_{17}^{*}}=\operatorname{Ind}_{N}^{U} \lambda^{A_{17}^{*}, 0,0}=\operatorname{Ind}_{H}^{U}\left(\sum_{A_{12} \in \mathbb{F}_{q}} \tilde{\chi}^{A_{17}^{*}, 0, A_{12}}\right) \\
= & \operatorname{Ind}_{H}^{U}\left(\sum_{A_{12} \in \mathbb{F}_{q}} \tilde{\chi}_{6, q^{4}}^{A_{17}^{*}, A_{12}}\right)=\sum_{A_{12} \in \mathbb{F}_{q}} \operatorname{Ind}_{H}^{U} \tilde{\chi}_{6, q^{4}}^{A_{17}^{*}, A_{12}}=\sum_{A_{12} \in \mathbb{F}_{q}} \chi_{6, q^{4}}^{A_{17}^{*}, A_{12}}
\end{aligned}
$$

4.14 Remark. The irreducible characters in the family $\mathfrak{F}_{4}$ in Proposition 4.12 are different for $p=2$, and the construction of these irreducible characters for can be found in [Le16. 3.3]. The irreducible characters in the other families are the same as these for $p>2$.

\section{Character table}

In this section, we determine the character table of the Sylow $p$-subgroup $U$ of ${ }^{3} D_{4}\left(q^{3}\right)$ when $p>2$. We use the notations as in Section 4 .

5.1 Proposition. The character table of $U$ for $p>2$ is the one in Table 4 
Table 4: Character table of $U$ for $p>2$

\begin{tabular}{|c|c|c|c|c|c|c|c|c|c|}
\hline & $I_{8}$ & $\begin{array}{l}x_{1}\left(t_{1}^{*}\right) \\
x_{6}\left(t_{6}\right)\end{array}$ & $\begin{array}{l}x_{1}\left(t_{1}^{*}\right) \\
\cdot x_{3}\left(\bar{t}_{3}^{*}\right)\end{array}$ & $\begin{array}{l}x_{2}\left(t_{2}^{*}\right) \\
\cdot x_{4}\left(t_{4}\right) \\
\cdot x_{5}\left(t_{5}\right)\end{array}$ & $\begin{array}{l}x_{2}\left(t_{2}^{*}\right) \\
\cdot x_{1}\left(t_{1}^{*}\right)\end{array}$ & $\begin{array}{l}x_{3}\left(t_{3}^{*}\right) \\
x_{5}\left(t_{5}\right)\end{array}$ & $x_{4}\left(t_{4}^{*}\right)$ & $x_{5}\left(t_{5}^{*}\right)$ & $x_{6}\left(t_{6}^{*}\right)$ \\
\hline$\chi_{\text {lin }}^{0,0}$ & 1 & 1 & 1 & 1 & 1 & 1 & 1 & 1 & 1 \\
\hline$\chi_{\operatorname{lin}}^{A_{12}^{*}, 0}$ & 1 & $\vartheta \pi_{q}\left(A_{12}^{*} t_{1}^{*}\right)$ & $\vartheta \pi_{q}\left(A_{12}^{*} t_{1}^{*}\right)$ & 1 & $\vartheta \pi_{q}\left(A_{12}^{*} t_{1}^{*}\right)$ & 1 & 1 & 1 & 1 \\
\hline$\chi_{l i n}^{0, A_{23}^{*}}$ & 1 & 1 & 1 & $\vartheta\left(A_{23}^{*} t_{2}^{*}\right)$ & $\vartheta\left(A_{23}^{*} t_{2}^{*}\right)$ & 1 & 1 & 1 & 1 \\
\hline$\chi_{l i n}^{A_{12}^{*}, A_{23}^{*}}$ & 1 & $\vartheta \pi_{q}\left(A_{12}^{*} t_{1}^{*}\right)$ & $\vartheta \pi_{q}\left(A_{12}^{*} t_{1}^{*}\right)$ & $\vartheta\left(A_{23}^{*} t_{2}^{*}\right)$ & $\begin{array}{l}\vartheta \pi_{q}\left(A_{12}^{*} t_{1}^{*}\right) \\
\vartheta\left(A_{23}^{*} t_{2}^{*}\right)\end{array}$ & 1 & 1 & 1 & 1 \\
\hline$\chi_{3, q}^{A_{13}^{*}, \bar{A}_{12}^{A_{13}^{*}}}$ & $q$ & * & * & 0 & 0 & $\begin{array}{l}\vartheta \pi_{q}\left(-A_{13}^{*} t_{3}^{*}\right) \\
\cdot q\end{array}$ & $q$ & $q$ & $q$ \\
\hline$\chi_{4, q^{3}}^{A_{15}^{*}, A_{23}}$ & $q^{3}$ & 0 & 0 & $*$ & 0 & 0 & $\begin{array}{l}\vartheta \pi_{q}\left(A_{15}^{*} t_{4}^{*}\right) \\
\cdot \vartheta \pi_{q}\left(A_{15}^{*} t_{4}^{* q^{2}}\right) \\
\cdot q^{3}\end{array}$ & $q^{3}$ & $q^{3}$ \\
\hline$\chi_{5, q^{3}}^{A_{16}^{*}, A_{23}, A_{13}}$ & $q^{3}$ & 0 & 0 & $*$ & 0 & $*$ & 0 & $\begin{array}{l}\vartheta\left(A_{16}^{*} t_{5}^{*}\right) \\
\cdot q^{3}\end{array}$ & $q^{3}$ \\
\hline$\chi_{6, q^{4}}^{A_{17}^{*}, A_{12}}$ & $q^{4}$ & * & 0 & 0 & 0 & 0 & 0 & 0 & $\begin{array}{l}\vartheta\left(A_{17}^{*} t_{6}^{*}\right) \\
\cdot q^{4}\end{array}$ \\
\hline
\end{tabular}

where the elements of the 1st column (i.e. the row headers) are the complete pairwise orthogonal irreducible characters of $U$, and more details can be seen in Proposition 4.12, e.g. $\bar{A}_{12}^{A_{13}^{*}} \in T^{A_{13}^{*}}$. The entries of the 1st row (i.e. the column headers) are all of the representatives of conjugacy classes of $U$, and more details can be seen in Proposition 3.11 e.g. $0 \neq \bar{t}_{3}^{*} \in T^{t_{1}^{*}}$. The $6 *$-values in the table are followed: 
The $(9,3)$-entry of Table 4 is

$$
\begin{aligned}
\chi_{6, q^{4}}^{A_{17}^{*}, A_{12}}\left(x_{1}\left(t_{1}^{*}\right) x_{6}\left(t_{6}\right)\right) & =\sum_{r_{3} \in \mathbb{F}_{q^{3}}} \vartheta \pi_{q}\left(A_{12} t_{1}^{*}+A_{17}^{*} \phi_{0}\left(-t_{1}^{*} r_{3}^{q^{2}+q}\right)+A_{17}^{*} t_{6}\right) \\
& =\vartheta \pi_{q}\left(A_{12} t_{1}^{*}+A_{17}^{*} t_{6}\right) \cdot \sum_{r_{3} \in \mathbb{F}_{q^{3}}} \vartheta\left(A_{17}^{*} \phi_{0}\left(-t_{1}^{*} r_{3}^{q^{2}+q}\right)\right),
\end{aligned}
$$

The $(8,5)$-entry of Table 4 is

$$
\begin{aligned}
\chi_{5, q^{3}}^{A_{16}^{*}, A_{23}, A_{13}}\left(x_{3}\left(t_{3}^{*}\right) x_{5}\left(t_{5}\right)\right) & =\sum_{r_{1} \in \mathbb{F}_{q^{3}}} \vartheta \pi_{q}\left(-A_{13} t_{3}^{*}+A_{16}^{*} \phi_{0}\left(t_{3}^{*} r_{1}^{q^{2}+q}\right)+A_{16}^{*} t_{5}\right) \\
& =\vartheta \pi_{q}\left(-A_{13} t_{3}^{*}+A_{16}^{*} t_{5}\right) \cdot \sum_{r_{1} \in \mathbb{F}_{q^{3}}} \vartheta\left(A_{16}^{*} \phi_{0}\left(t_{3}^{*} r_{1}^{q^{2}+q}\right)\right),
\end{aligned}
$$

The $(8,7)$-entry of Table 4 is

$$
\begin{aligned}
& \chi_{5, q^{3}}^{A_{16}^{*}, A_{23}, A_{13}}\left(x_{2}\left(t_{2}^{*}\right) x_{4}\left(t_{4}\right) x_{5}\left(t_{5}\right)\right) \\
= & \sum_{r_{1} \in \mathbb{F}_{q^{3}}} \vartheta \pi_{q}\left(A_{23} t_{2}^{*}+A_{13} r_{1} t_{2}^{*}+A_{16}^{*}\left(\phi_{0}\left(r_{1} t_{4}^{q}\right)-t_{2}^{*} r_{1}^{q^{2}+q+1}\right)+A_{16}^{*} t_{5}\right) \\
= & \vartheta\left(A_{23} t_{2}^{*}+A_{16}^{*} t_{5}\right) \cdot \sum_{r_{1} \in \mathbb{F}_{q^{3}}} \vartheta \pi_{q}\left(A_{13} r_{1} t_{2}^{*}+A_{16}^{*}\left(\phi_{0}\left(r_{1} t_{4}^{q}\right)-t_{2}^{*} r_{1}^{q^{2}+q+1}\right)\right),
\end{aligned}
$$

The $(7,5)$-entry of Table 4 is

$$
\begin{aligned}
& \chi_{4, q^{3}}^{A_{15}^{*}, A_{23}}\left(x_{2}\left(t_{2}^{*}\right) x_{4}\left(t_{4}\right) x_{5}\left(t_{5}\right)\right) \\
= & \sum_{r_{1} \in \mathbb{F}_{q^{3}}} \vartheta \pi_{q}\left(A_{23} t_{2}^{*}+A_{15}^{*}\left(t_{4}-t_{2}^{*} r_{1}^{q+1}\right)+A_{15}^{*}\left(t_{4}^{q^{2}}-t_{2}^{*} r_{1}^{q^{2}+1}\right)\right) \\
= & \vartheta\left(A_{23} t_{2}^{*}\right) \cdot \sum_{r_{1} \in \mathbb{F}_{q^{3}}} \vartheta \pi_{q}\left(A_{15}^{*}\left(t_{4}-t_{2}^{*} r_{1}^{q+1}\right)+A_{15}^{*}\left(t_{4}^{q^{2}}-t_{2}^{*} r_{1}^{q^{2}+1}\right)\right),
\end{aligned}
$$

The (6,3)-entry of Table 4 is

$$
\chi_{3, q}^{A_{13}^{*}, \bar{A}_{12}^{A_{13}^{*}}}\left(x_{1}\left(t_{1}^{*}\right) x_{6}\left(t_{6}\right)\right)=\sum_{r_{2} \in \mathbb{F}_{q}} \vartheta \pi_{q}\left(\left(\bar{A}_{12}^{A_{13}^{*}}-A_{13}^{*} r_{2}\right) t_{1}^{*}\right),
$$

The $(6,4)$-entry of Table 4 is

$$
\chi_{3, q}^{A_{13}^{*}, \bar{A}_{12}^{A_{13}^{*}}}\left(x_{1}\left(t_{1}^{*}\right) x_{3}\left(\bar{t}_{3}^{*}\right)\right)=\sum_{r_{2} \in \mathbb{F}_{q}} \vartheta \pi_{q}\left(\left(\bar{A}_{12}^{A_{13}^{*}}-A_{13}^{*} r_{2}\right) t_{1}^{*}-\bar{t}_{3}^{*} A_{13}^{*}\right) .
$$

Proof. Let $x:=x\left(t_{1}, t_{2}, t_{3}, t_{4}, t_{5}, t_{6}\right) \in U$.

(1) Calculate the values of $\chi_{6, q^{4}}^{A_{17}^{*}, A_{12}}$ for all $A_{17}^{*} \in \mathbb{F}_{q}^{*}$ and $A_{12} \in \mathbb{F}_{q^{3}}$.

We use the notations of (5) of Proposition 4.12, then

$$
\begin{aligned}
\chi_{6, q^{4}}^{A_{17}^{*}, A_{12}}(x) & =\left(\operatorname{Ind}_{H}^{U} \tilde{\chi}_{6, q^{4}}^{A_{17}^{*}, A_{12}}\right)(x)=\frac{1}{|H|} \sum_{\substack{g \in U \\
g \cdot x \cdot g^{-1} \in H}} \tilde{\chi}_{6, q^{4}}^{A_{17}^{*}, A_{12}}\left(g \cdot x \cdot g^{-1}\right) \\
& =\frac{1}{|H|} \sum_{\substack{g \in U \\
g \cdot x \cdot g^{-1} \in H}} \bar{\chi}_{6, q^{4}}^{A_{17}^{*}, A_{12}}\left(X_{4} X_{5} \cdot\left(g x g^{-1}\right)\right) .
\end{aligned}
$$


Thus,

$$
\begin{aligned}
& \chi_{6, q^{4}}^{A_{17}^{*}, A_{12}}\left(x_{1}\left(t_{1}^{*}\right) x_{3}\left(\bar{t}_{3}^{*}\right)\right)=\chi_{6, q^{4}}^{A_{17}^{*}, A_{12}}\left(x_{2}\left(t_{2}^{*}\right) x_{4}\left(t_{4}\right) x_{5}\left(t_{5}\right)\right) \\
= & \chi_{6, q^{4}}^{A_{17}^{*}, A_{12}}\left(x_{2}\left(t_{2}^{*}\right) x_{1}\left(t_{1}^{*}\right)\right)=\chi_{6, q^{4}}^{A_{17}^{*}, A_{12}}\left(x_{3}\left(t_{3}^{*}\right) x_{5}\left(t_{5}\right)\right) \stackrel{g x g^{-1} \notin H}{=}{ }^{g} 0 .
\end{aligned}
$$

Then

$$
\begin{aligned}
& \chi_{6, q^{4}}^{A_{17}^{*}, A_{12}}\left(x_{4}\left(t_{4}\right) x_{5}\left(t_{5}\right) x_{6}\left(t_{6}\right)\right) \\
= & \frac{1}{|H|} \sum_{\substack{g:=x\left(r_{1}, r_{2}, r_{3}, r_{4}, r_{5}, r_{6}\right) \in U \\
g \cdot x_{4}\left(t_{4}\right) x_{5}\left(t_{5}\right) x_{6}\left(t_{6}\right) \cdot g^{-1} \in H}} \tilde{\chi}_{6, q^{4}}^{A_{17}^{*}, A_{12}}\left(g \cdot x_{4}\left(t_{4}\right) x_{5}\left(t_{5}\right) x_{6}\left(t_{6}\right) \cdot g^{-1}\right) \\
= & \frac{1}{|H|} \sum_{\substack{r_{1}, r_{3}, r_{4} \in \mathbb{F}_{q 3} \\
r_{2}, r_{5}, r_{6} \in \mathbb{F}_{q}}}^{\tilde{\chi}_{6, q^{4}}^{A_{17}^{*}, A_{12}}\left(x_{4}\left(t_{4}\right) x_{5}\left(t_{5}+\phi_{0}\left(r_{1} t_{4}^{q}\right)\right)\right.} \\
= & \frac{1}{|H|} \sum_{\substack{r_{1}, r_{3}, r_{4} \in \mathbb{F}_{q^{3}} \\
r_{2}, r_{5}, r_{6} \in \mathbb{F}_{q}}} \bar{\chi}_{6, q^{4}}^{A_{17}^{*}, A_{12}}\left(\bar{x}_{6}\left(t_{6}+r_{2} t_{5}+\phi_{0}\left(r_{1} r_{2} t_{4}^{q}\right)+\phi_{0}\left(r_{1} r_{2} t_{4}^{q}\right)+\phi_{0}\left(r_{3} t_{4}^{q}\right)\right)\right) \\
= & \frac{1}{q^{3}} \sum_{\substack{r_{1}, r_{3} \in \mathbb{F}_{q^{3}} \\
r_{2} \in \mathbb{F}_{q}}} \bar{\chi}_{6, q^{4}}^{A_{17}^{*}, A_{12}}\left(\bar{x}_{6}\left(t_{6}+r_{2} t_{5}+\phi_{0}\left(r_{1} r_{2} t_{4}^{q}\right)+\phi_{0}\left(r_{3} t_{4}^{q}\right)\right)\right),
\end{aligned}
$$

So,

$\chi_{6, q^{4}}^{A_{17}^{*}, A_{12}}\left(I_{8}\right)=q^{4}, \chi_{6, q^{4}}^{A_{17}^{*}, A_{12}}\left(x_{4}\left(t_{4}^{*}\right)\right)=\chi_{6, q^{4}}^{A_{17}^{*}, A_{12}}\left(x_{5}\left(t_{5}^{*}\right)\right)=0, \chi_{6, q^{4}}^{A_{17}^{*}, A_{12}}\left(x_{6}\left(t_{6}^{*}\right)\right)=q^{4} \cdot \vartheta\left(A_{17}^{*} t_{6}^{*}\right)$.

Then

$$
\begin{aligned}
\chi_{6, q^{4}}^{A_{17}^{*}, A_{12}}\left(x_{1}\left(t_{1}^{*}\right) x_{6}\left(t_{6}\right)\right) & =\frac{1}{|H|} \sum_{\substack{g \in U \\
g \cdot x_{1}\left(t_{1}^{*}\right) x_{6}\left(t_{6}\right) \cdot g^{-1} \in H}} \tilde{\chi}_{6, q^{4}}^{A_{17}^{*}, A_{12}}\left(g \cdot x_{1}\left(t_{1}^{*}\right) x_{6}\left(t_{6}\right) \cdot g^{-1}\right) \\
g:=x\left(r_{1}, r_{2}, r_{3}, r_{4}, r_{5}, r_{6}\right) & \frac{1}{|H|} \sum_{\substack{r_{2}=0 \\
r_{1}, r_{3}, r_{4} \in \mathbb{F}_{q} \\
r_{5}, r_{6} \in \mathbb{F}_{q}}} \bar{\chi}_{6, q^{4}}^{A_{17}^{*}, A_{12}}\left(\bar{x}_{1}\left(t_{1}^{*}\right) \bar{x}_{6}\left(t_{6}+\phi_{0}\left(-t_{1}^{*} r_{3}^{q^{2}+q}\right)\right)\right) \\
& =\sum_{r_{3} \in \mathbb{F}_{q^{3}}} \bar{\chi}_{6, q^{4}}^{A_{17}^{*}, A_{12}}\left(\bar{x}_{1}\left(t_{1}^{*}\right) \bar{x}_{6}\left(t_{6}+\phi_{0}\left(-t_{1}^{*} r_{3}^{q^{2}+q}\right)\right)\right) \\
& =\vartheta \pi_{q}\left(A_{12} t_{1}^{*}+A_{17}^{*} t_{6}\right) \cdot \sum_{r_{3} \in \mathbb{F}_{q^{3}}} \vartheta\left(A_{17}^{*} \phi_{0}\left(-t_{1}^{*} r_{3}^{q^{2}+q}\right)\right) .
\end{aligned}
$$

Thus all the values of $\chi_{6, q^{4}}^{A_{17}^{*}, A_{12}}$ are obtained.

(2) Calculate the values of $\chi_{3, q}^{A_{13}^{*}, \bar{A}_{12}^{A_{13}^{*}}}$ for all $A_{13}^{*} \in \mathbb{F}_{q^{3}}^{*}$ and $\bar{A}_{12}^{A_{13}^{*}} \in T^{A_{13}^{*}}$.

We use the notations of (2) of Proposition 4.12. For $x=x\left(t_{1}, t_{2}^{*}, t_{3}, t_{4}, t_{5}, t_{6}\right) \in U$ with $0 \neq t_{2}^{*} \in \mathbb{F}_{q}^{*}$, we have

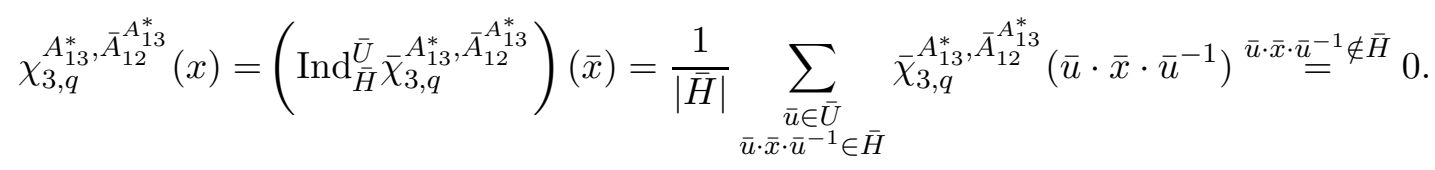


If $x=x\left(t_{1}, 0, t_{3}, t_{4}, t_{5}, t_{6}\right) \in U$, we have

$$
\begin{aligned}
& \chi_{3, q}^{A_{13}^{*}, \bar{A}_{12}^{A_{13}^{*}}}\left(x\left(t_{1}, 0, t_{3}, t_{4}, t_{5}, t_{6}\right)\right)=\left(\operatorname{Ind}_{\bar{H}}^{\bar{U}} \bar{\chi}_{3, q}^{A_{13}^{*}, \bar{A}_{12}^{A_{13}^{*}}}\right)\left(\bar{x}_{1}\left(t_{1}\right) \bar{x}_{3}\left(t_{3}\right)\right) \\
& =\frac{1}{|\bar{H}|} \sum_{\begin{array}{c}
\bar{u}:=\bar{x}_{2}\left(r_{2}\right) \bar{x}_{1}\left(r_{1}\right) \bar{x}_{3}\left(r_{3}\right) \in \bar{U} \\
\bar{u} \cdot \bar{x}_{1}\left(t_{1}\right) \bar{x}_{3}\left(t_{3}\right) \cdot \bar{u}^{-1} \in \bar{H}
\end{array}} \bar{\chi}_{3, q}^{A_{13}^{*}, \bar{A}_{12}^{A_{13}^{*}}}\left(\bar{u} \cdot \bar{x}_{1}\left(t_{1}\right) \bar{x}_{3}\left(t_{3}\right) \cdot \bar{u}^{-1}\right) \\
& =\frac{1}{|\bar{H}|} \sum_{\substack{r_{1}, r_{3} \in \mathbb{F}_{q^{3}} \\
r_{2} \in \mathbb{F}_{q}}} \bar{\chi}_{3, q}^{A_{13}^{*}, \bar{A}_{12}^{A_{13}^{*}}}\left(\bar{x}_{1}\left(t_{1}\right) \bar{x}_{3}\left(t_{3}+r_{2} t_{1}\right)\right)
\end{aligned}
$$

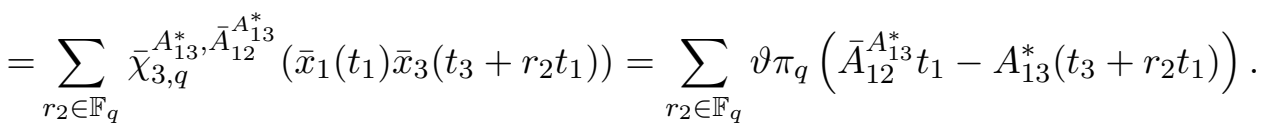

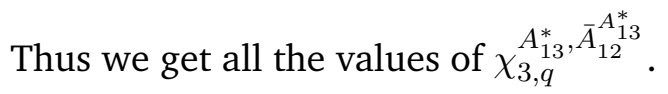

Similarly, we calculate all of the other values of the character table.

In the paper, $p$ is an odd prime. By Propositions 3.11, 4.12 and 5.1, we obtain the number of the conjugacy classes, and determine the numbers of the complex irreducible characters of degree $q^{c}$ with $c \in \mathbb{N}$. T. Le [Le16] counted the number of irreducible characters for all primes $p$.

5.2 Corollary. Let \#Irr be the number of irreducible characters of the Sylow p-subgroup $U$ of ${ }^{3} D_{4}\left(q^{3}\right)$, and \# $\operatorname{Irr}_{c}$ be the number of irreducible characters of $U$ of dimension $q^{c}$ with $c \in \mathbb{N}$, then

$$
\begin{array}{ll}
\# \operatorname{Irr}_{4}=q^{4}-q^{3} & =(q-1)^{4}+3(q-1)^{3}+3(q-1)^{2}+(q-1), \\
\# \operatorname{Irr}_{3}=q^{5}-q & =(q-1)^{5}+5(q-1)^{4}+10(q-1)^{3}+10(q-1)^{2}+4(q-1), \\
\text { Irr }_{1}=q^{5}-q^{2} & =(q-1)^{5}+5(q-1)^{4}+10(q-1)^{3}+9(q-1)^{2}+3(q-1), \\
\# \operatorname{Irr}_{0}=q^{4} & =(q-1)^{4}+4(q-1)^{3}+6(q-1)^{2}+4(q-1)+1,
\end{array}
$$

and

$$
\begin{aligned}
\# \operatorname{Irr} & =\#\{\text { Irreducible Characters of } U\} \\
& =\#\{\text { Conjugacy Classes of } U\} \\
& =2 q^{5}+2 q^{4}-q^{3}-q^{2}-q \\
& =2(q-1)^{5}+12(q-1)^{4}+27(q-1)^{3}+28(q-1)^{2}+12(q-1)+1 .
\end{aligned}
$$

5.3 Remark. We also consider the analogue of Higman's conjecture, Lehrer's conjecture and Isaacs' conjecture of $U_{n}(q)$ for $U$. By[5.2] the conjectures are true for $U$.

\section{Acknowledgements}

This paper is part of my $\mathrm{PhD}$ thesis [Sun17] at the University of Stuttgart, Germany, so I am deeply grateful to my supervisor Richard Dipper. I also would like to thank Markus Jedlitschky, Mathias Werth and Yichuan Yang for the helpful discussions and valuable suggestions.

\section{References}

[Car72] Roger W. Carter. Simple groups of Lie type. John Wiley \& Sons, New York, 1972. 
[CR81] Charles W. Curtis and Irving Reiner. Methods of representation theory: with applications to finite groups and orders, volume I. John Wiley \& Sons, Inc., New York, 1981.

[GLM17] Simon M. Goodwin, Tung Le, and Kay Magaard. The generic character table of a Sylow $p$-subgroup of a finite Chevalley group of type $D_{4}$. Comm. Algebra, 45(12):5158-5179, 2017.

[GLMP16] Simon M. Goodwin, Tung Le, Kay Magaard, and Alessandro Paolini. Constructing characters of Sylow p-subgroups of finite Chevalley groups. J. Algebra, 468:395439, 2016.

[GMR14] Simon M. Goodwin, Peter Mosch, and Gerhard Röhrle. Calculating conjugacy classes in Sylow $p$-subgroups of finite Chevalley groups of rank six and seven. LMS J. Comput. Math., 17(1):109-122, 2014.

[GMR16] Simon M. Goodwin, Peter Mosch, and Gerhard Röhrle. On the coadjoint orbits of maximal unipotent subgroups of reductive groups. Transformation Groups, 21(2):399-426, 2016.

[GR09] Simon M. Goodwin and Gerhard Röhrle. Calculating conjugacy classes in Sylow p-subgroups of finite Chevalley groups. J. Algebra, 321(11):3321-3334, 2009.

[Hig60] Graham Higman. Enumerating p-groups. I. Inequalities. Proc. London Math. Soc. (3), 10:24-30, 1960.

[HLM11] Frank Himstedt, Tung Le, and Kay Magaard. Characters of the Sylow $p$-subgroups of the Chevalley groups $D_{4}\left(p^{n}\right)$. J. Algebra, 332:414-427, 2011.

[HLM16] Frank Himstedt, Tung Le, and Kay Magaard. On the characters of the Sylow $p$ subgroups of untwisted Chevalley groups $Y_{n}\left(p^{a}\right)$. LMS J. Comput. Math., 19(2):303359, 2016.

[Hum78] James E. Humphreys. Introduction to Lie algebras and representation theory, volume 9 of Graduate Texts in Mathematics. Springer-Verlag, New York-Berlin, 1978. Second printing, revised.

[Isa95] I. M. Isaacs. Characters of groups associated with finite algebras. J. Algebra, 177(3):708-730, 1995.

[Isa07] I. M. Isaacs. Counting characters of upper triangular groups. J. Algebra, 315(2):698719, 2007.

[Jed13] Markus Jedlitschky. Decomposing André-Neto supercharacters of Sylow p-subgroups of Lie type D. PhD thesis, Universität Stuttgart, 2013.

[Le16] Tung Le. Irreducible characters of Sylow $p$-subgroups of the Steinberg triality groups ${ }^{3} D_{4}\left(p^{3 m}\right)$. Bull. Iranian Math. Soc., 42(5):1279-1291, 2016.

[Leh74] G. I. Lehrer. Discrete series and the unipotent subgroup. Compositio Math., 28:9-19, 1974.

[PS15] Igor Pak and Andrew Soffer. On Higman's $k\left(U_{n}(q)\right)$ conjecture. preprint arXiv: 1507.00411v1, 2015.

[Sun17] Yujiao Sun. Supercharacter theories for Sylow p-subgroups ${ }^{3} D_{4}^{\text {syl }}\left(q^{3}\right), G_{2}^{\text {syl }}(q)$ and ${ }^{2} G_{2}^{s y l}\left(3^{2 m+1}\right) . \mathrm{PhD}$ thesis, Universität Stuttgart, 2017. 
[VLA03] Antonio Vera-López and J. M. Arregi. Conjugacy classes in unitriangular matrices. Linear Algebra Appl., 370:85-124, 2003. 